\title{
Asymptotic behavior and rigidity results for symmetric solutions of the elliptic system $\Delta u=W_{u}(u)$
}

\author{
Nicholas D. Alikakos And Giorgio Fusco
}

\begin{abstract}
We study symmetric vector minimizers of the Allen-Cahn energy $J(u)=\int\left(\frac{1}{2}|\nabla u|^{2}+W(u)\right) d x$ and establish various results concerning their structure and their asymptotic behavior in unbounded domains.
\end{abstract}

Mathematics Subject Classification (2010): 35J47 (primary); 35J50, 35J20 (secondary).

\section{Introduction}

The problem of describing the structure of bounded solutions $u: \Omega \rightarrow \mathbb{R}^{m}$ of the equation

$$
\Delta u=f(u), \quad \text { for } \quad x \in \Omega
$$

where $f: \mathbb{R}^{m} \rightarrow \mathbb{R}^{m}$ is a smooth map and $\Omega \subset \mathbb{R}^{n}$ is a smooth domain that can be bounded or unbounded and may also enjoy symmetry properties, is a difficult and important problem which has attracted the interest of many authors in the last twentyfive years, see $[8,9,11,17]$ just to mention a few. Questions concerning monotonicity, symmetry and asymptotic behavior are the main objectives of these investigations. Most of the existing literature concerns the scalar case $m=1$ where a systematic use of the maximum principle and its consequences are the main tools at hand. For the vector case $m \geq 2$ we mention the works [10] and [18]

The first author was partially supported through the project PDEGE Partial Differential Equations Motivated by Geometric Evolution, co-financed by the European Union European Social Fund (ESF) and national resources, in the framework of the program Aristeia of the Operational Program Education and Lifelong Learning of the National Strategic Reference Framework (NSRF). The research of N. Alikakos has been co-financed by the European Union European Social Fund (ESF) and Greek national funds through the Operational Program Education and Lifelong Learning of the National Strategic Reference Framework (NSRF) - Research Funding Program: THALES

Received February 10, 2014; accepted September 17, 2014.

Published online February 2016. 
where the control of the asymptotic behavior of solutions was basic for proving existence. In this paper we are interested in the case where $f(u)=W_{u}(u)$ is the gradient of a potential $W: \mathbb{R}^{m} \rightarrow \mathbb{R}$ and $u$ is a minimizer for the action functional $\int\left(\frac{1}{2}|\nabla v|^{2}+W(v)\right)$, the Allen-Cahn energy, in the following sense:

Definition 1.1. A map $u \in C^{2}\left(\Omega ; \mathbb{R}^{m}\right) \cap L^{\infty}\left(\Omega ; \mathbb{R}^{m}\right)$, with $\Omega \subset \mathbb{R}^{n}$ an open set, is said to be a minimizer or minimal if for each bounded open lipshitz set $\Omega^{\prime} \subset \Omega$ it results

$$
J_{\Omega^{\prime}}(u)=\min _{v \in W_{0}^{1,2}\left(\Omega^{\prime} ; \mathbb{R}^{m}\right)} J_{\Omega^{\prime}}(u+v), \quad J_{\Omega^{\prime}}(v)=\int_{\Omega^{\prime}}\left(\frac{1}{2}|\nabla v|^{2}+W(v)\right),
$$

that is $\left.u\right|_{\Omega^{\prime}}$ is an absolute minimizer in the set of $W^{1,2}\left(\Omega^{\prime} ; \mathbb{R}^{m}\right)$ maps which coincide with $u$ on $\partial \Omega^{\prime}$.

Clearly if $u: \Omega \rightarrow \mathbb{R}^{m}$ is minimal then it is a solution of the Euler-Lagrange equation associated to the functional $J_{\Omega^{\prime}}$ which is the vector Allen-Cahn equation

$$
\Delta u=W_{u}(u), \quad \text { for } \quad x \in \Omega .
$$

We will work in the context of reflection symmetries. Our main results are Theorem 1.4 on the asymptotic behavior of symmetric minimizers and Theorem 1.7 and Theorem 1.8 on the rigidity of symmetric minimizers. By rigidity we mean that, under suitable assumptions, a symmetric minimizer $u: \mathbb{R}^{n} \rightarrow \mathbb{R}^{m}$ must in effect depend on a number of variables $k<n$ strictly less than the dimension $n$ of the domain space. These theorems, in the symmetric setting, are vector counterparts of analogous results which are well known in the scalar case $m=1[7,13]$. However in the vector case there is more structure as we explain after the statement of Theorem 1.7.

We let $G$ be a reflection group acting both on the domain space $\Omega \subseteq \mathbb{R}^{n}$ and on the target space $\mathbb{R}^{m}$. We assume that $W: \mathbb{R}^{m} \rightarrow \mathbb{R}$ is a nonnegative $C^{3}$ potential such that:

$\mathbf{H}_{1} W$ is symmetric with respect to $G: W(g u)=W(u)$, for $g \in G$, and $u \in \mathbb{R}^{m}$.

For Theorem 1.4 and Theorem 1.7, $G=S$, where $S$ denotes the group of order 2 generated by the reflection $\mathbb{R}^{d} \ni z \mapsto \hat{z} \in \mathbb{R}^{d}$ in the plane $\left\{z_{1}=0\right\}$ :

$$
\hat{z}=\left(-z_{1}, z_{2}, \ldots, z_{d}\right), \quad \text { for } \quad d=n, m .
$$

In this case the symmetry of $W$ is expressed by $W(\hat{u})=W(u)$, for $u \in \mathbb{R}^{m}$. For Theorem 1.8, $G=T$, where $T$ denotes the group of order 6 of the symmetries of the equilateral triangle. $T$ is generated by the reflection $\gamma$ through the hyperplane $\left\{z \in \mathbb{R}^{d}: z_{2}=0\right\}$ and by the reflections $\gamma_{ \pm}$through the hyperplane $\left\{z \in \mathbb{R}^{d}: z_{2}=\right.$ $\left.\pm \sqrt{3} z_{1}\right\}$. We let $F \subset \mathbb{R}^{d}$, for $d=n$ or $d=m$ be a fundamental region [19] for the action of $G$ on $\mathbb{R}^{d}$. If $G=S$ we take $F=\mathbb{R}_{+}^{d}=\left\{z \in \mathbb{R}^{d}: z_{1}>0\right\}$. If $G=T$ we take $F=\left\{z \in \mathbb{R}^{d}: 0<z_{2}<\sqrt{3} z_{1}, z_{1}>0\right\}$. 
$\mathbf{H}_{2}$ There exists $a \in \bar{F} \subset \mathbb{R}^{m}$ such that:

$$
0=W(a) \leq W(u), \quad \text { for } \quad u \in \bar{F} .
$$

Moreover $a$ is nondegenerate in the sense that the quadratic form $D^{2} W(a)(z, z)$ is positive definite.

In the symmetric setting we assume minimality in the class of symmetric variations:

Definition 1.2. Assume that $\Omega \subset \mathbb{R}^{n}$ and $u \in C^{2}\left(\Omega ; \mathbb{R}^{m}\right) \cap L^{\infty}\left(\Omega ; \mathbb{R}^{m}\right)$ are symmetric:

$$
\begin{aligned}
& x \in \Omega \Rightarrow g x \in \Omega, \text { for } g \in G, \\
& u(g x)=g u(x), \text { for } g \in G, \text { and } x \in \Omega .
\end{aligned}
$$

Then $u$ is said to be a symmetric minimizer if for each bounded open symmetric lipschitz set $\Omega^{\prime} \subset \Omega$ and for each bounded symmetric $v \in W_{0}^{1,2}\left(\Omega^{\prime} ; \mathbb{R}^{m}\right)$ it results

$$
J_{\Omega^{\prime}}(u) \leq J_{\Omega^{\prime}}(u+v)
$$

In the following by a minimizer we will always mean a symmetric minimizer in the sense of the definition above. We set

$$
\mathcal{Z}=\left\{z \in \mathbb{R}^{m}: z \neq a, W(z)=0\right\}
$$

Theorem 1.3. Assume $G=S$ and assume that $W$ satisfies $\mathbf{H}_{1}-\mathbf{H}_{2}$. Assume that $\Omega \subseteq \mathbb{R}^{n}$ is convex-symmetric in the sense that

$$
x=\left(x_{1}, x_{2}, \ldots, x_{n}\right) \in \Omega \Rightarrow\left(t x_{1}, x_{2} \ldots, x_{n}\right) \in \Omega, \text { for }|t| \leq 1 .
$$

Let $u: \Omega \rightarrow \mathbb{R}^{m}$ be a minimizer and assume that there are $\delta>0$ and $d_{0} \geq 0$ such that

$$
|u(x)-z|>\delta, \text { for } z \in \mathcal{Z}, d\left(x, \partial \Omega^{+}\right) \geq d_{0}, \text { and } x \in \Omega^{+}
$$

where $\Omega^{+}=\left\{x \in \Omega: x_{1}>0\right\}$. Assume moreover that

$$
|u|+|\nabla u| \leq M, \text { for } x \in \Omega,
$$

for some $M>0$.

Then there exist $k_{0}, K_{0}>0$ such that

$$
|u-a| \leq K_{0} e^{-k_{0} d\left(x, \partial \Omega^{+}\right)}, \text {for } x \in \Omega^{+} .
$$

Proof. From (1.8) and (1.9) it follows that the restriction of the minimizer $u$ to the subset $\left\{x \in \Omega: x_{1}>d_{0}\right\}$ satisfies the assumptions of Theorem 1.2 in [15], which implies the estimate (1.10). 
For example, if $\Omega=\left\{x \in \mathbb{R}^{2}: x_{2}>0\right\},(1.10)$ yields

$$
|u-a| \leq K_{0} e^{-k_{0} \min \left\{x_{1}, x_{2}\right\}}, \quad \text { for } x \in \Omega^{+} .
$$

This implies $\lim _{t \rightarrow+\infty} u(t z)=a$ for each unit vector $z \in \Omega^{+}$but does not imply $\lim _{x_{1} \rightarrow+\infty} u\left(x_{1}, x_{2}\right)=a$ for fixed $x_{2}>0$ or $\lim _{x_{2} \rightarrow+\infty} u\left(x_{1}, x_{2}\right)=a$ for fixed $x_{1}>0$.

Examples of minimizers that satisfy the hypothesis of Theorem 1.3 are provided by the entire equivariant solutions of $(1.3)$ constructed in $[3,5,14]$. The gradient bound in (1.9) is a consequence of the smoothness of $\Omega$ or, as in the case of the entire solutions referred to above, it follows from the fact that $u$ in Theorem 1.3 is the restriction to a non smooth set $\Omega$ of a smooth map defined in $\mathbb{R}^{n}$.

We denote by $C_{S}^{\operatorname{Exp}}\left(\bar{\Omega}, \mathbb{R}^{m}\right)$ the set of lipschitz symmetric maps $v: \bar{\Omega} \rightarrow \mathbb{R}^{m}$ that satisfy the bounds

$$
\begin{aligned}
& \|v\|_{C^{0,1}\left(\bar{\Omega}, \mathbb{R}^{m}\right)} \leq M, \\
& |v-a|+|\nabla v| \leq K_{0} e^{-k_{0} d\left(x, \partial \Omega^{+}\right)}, \quad \text { for } \quad x \in \Omega^{+} .
\end{aligned}
$$

We remark that from (1.10) and elliptic regularity, after redefining $k_{0}$ and $K_{0}$ if necessary, we have

$$
u \in C_{S}^{\operatorname{Exp}}\left(\bar{\Omega}, \mathbb{R}^{m}\right),
$$

for the minimizer in Theorem 1.3.

Theorem 1.4. Assume $W, \Omega$ and $u: \Omega \rightarrow \mathbb{R}^{m}$ as in Theorem 1.3. Assume moreover that

$\mathbf{H}_{3}$ The problem

$$
\begin{cases}u^{\prime \prime}=W_{u}(u) & \text { for } s \in \mathbb{R} \\ u(-s)=\hat{u}(s) & \text { for } s \in \mathbb{R} \\ \lim _{s \rightarrow+\infty} u(s)=a, & \end{cases}
$$

has a unique solution $\bar{u}: \mathbb{R} \rightarrow \mathbb{R}^{m}$.

$\mathbf{H}_{4}$ The operator $T: D(T) \rightarrow L^{2}\left(\mathbb{R}, \mathbb{R}^{m}\right)$ defined by

$$
D(T)=W_{S}^{2,2}\left(\mathbb{R}, \mathbb{R}^{m}\right), \quad T v=-v^{\prime \prime}+W_{u u}(\bar{u}) v,
$$

where $W_{S}^{2,2}\left(\mathbb{R}, \mathbb{R}^{m}\right) \subset W^{2,2}\left(\mathbb{R}, \mathbb{R}^{m}\right)$ is the subspace of symmetric maps, has a trivial kernel.

Then there exist $k, K>0$, depending only on $W$ and $M$, such that

$$
\left|u(x)-\bar{u}\left(x_{1}\right)\right| \leq K e^{-k d(x, \partial \Omega)}, \quad \text { for } \quad x \in \Omega .
$$


Remark 1.5. A significant feature of Theorem 1.4 is that the estimate (1.15) is valid, with $k, K$ independent of $\Omega$, for bounded or unbounded domains and in particular for domains bounded in the $x_{1}$ direction.

Remark 1.6. The map $\bar{u}$ in assumption $\mathbf{H}_{3}$ can be characterized as a minimizer of the functional $\mathcal{A}(v)=\int_{\mathbb{R}}\left(\frac{1}{2}\left|v^{\prime}\right|^{2}+W(u)\right) d s$ in the set of symmetric $W_{\text {loc }}^{1,2}\left(\mathbb{R}, \mathbb{R}^{m}\right)$ maps that satisfy

$$
\lim _{s \rightarrow+\infty} v(s)=a .
$$

Theorem 1.7. Assume that $\Omega=\mathbb{R}^{n}$ and that $W$ and $u: \mathbb{R}^{n} \rightarrow \mathbb{R}^{m}$ are as in Theorem 1.4. Then $u$ is unidimensional:

$$
u(x)=\bar{u}\left(x_{1}\right), x \in \mathbb{R}^{n} .
$$

From $[3,5]$ and [14], we know that given a finite reflection group $G$, provided $W$ is invariant under $G$, there exists a $G$-equivariant solutions $u: \mathbb{R}^{n} \rightarrow \mathbb{R}^{m}$ of the system (1.3). It is natural to ask about the asymptotic behavior of these solutions. In particular, given a unit vector $v=\left(v_{1}, \ldots, v_{n}\right) \in \mathbb{R}^{n}$ one may wonder about the existence of the limit

$$
\lim _{\lambda \rightarrow+\infty} u\left(x^{\prime}+\lambda v\right)=\tilde{u}\left(x^{\prime}\right)
$$

where $x^{\prime}$ is the projection of $x=x^{\prime}+\lambda \nu$ on the hyperplane orthogonal to $v$. One can conjecture that this limit does indeed exist and that $\tilde{u}$ is a solution of the same system equivariant with respect to the subgroup $G_{v} \subset G$ that leave $v$ fixed, i.e. the stabilizer of $v$. In [3,5] and [14] an exponential estimate analogous to (1.10) in Theorem 1.3 was established. This gives a positive answer to this conjecture for the case where $v$ is inside the set $D=\operatorname{Int} \cup_{g \in G_{a}} g \bar{F} \subset \mathbb{R}^{n}$. Here $F$ is a fundamental region for the action of $G$ on $\mathbb{R}^{d}$, for $d=n, m$ and $G_{a} \subset G$ is the subgroup that leave $a$ fixed. Under the assumptions $\mathbf{H}_{3}$ and $\mathbf{H}_{4}$ Theorem 1.4 goes one step forward and shows that the conjecture is true when $v$ belongs to the interior of one of the walls of the set $D$ above and $G_{v}$ is the subgroup of order two generated by the reflection with respect to that wall. In the proof of Theorem 1.4 the estimate (1.10) is basic. Once the exponential estimate in Theorem 1.4 is established, we conjecture that, under assumptions analogous to $\mathbf{H}_{3}$ and $\mathbf{H}_{4}$, the approach developed in the proof of Theorem 1.4 can be used to handle the case where $v$ belongs to the intersection of two walls of $D$. We also expect that, under the assumption that at each step $\tilde{u}$ is unique and hyperbolic, the process can be repeated to establish the whole hierarchy of limits corresponding to all possible choice of $v$ and $\tilde{u}$ is always a solution of the system equivariant with respect to the subgroup $G_{v}$. This program is motivated by the analogy between equivariant connection maps and minimal cones [4].

Theorem 1.8 below gives an example, in the diffuse interface set-up, that corresponds to a minimal cone that splits into a triod and a hyperplane [22]. Theorem 1.8 concerns minimizers equivariant with respect to the symmetry group $T$ of the 
equilateral triangle. We can imagine that $T=G_{v}$ for some $v$ that belongs to the intersection of two walls of $D$. The following assumptions $\mathbf{H}_{3}^{\prime}$ and $\mathbf{H}_{4}^{\prime}$, in the case at hand where $G=T$, correspond to the assumption $\mathbf{H}_{3}$ and $\mathbf{H}_{4}$ in Theorem 1.4. We assume that

$$
a=(1,0, \ldots, 0) \in \mathbb{R}^{m} \text {. }
$$

$\mathbf{H}_{3}^{\prime}$ The problem

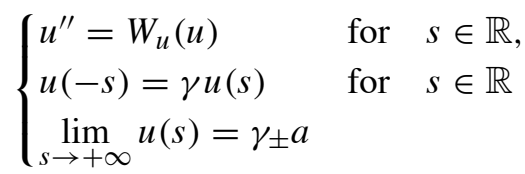

has a unique solution $\bar{u}: \mathbb{R} \rightarrow \mathbb{R}^{m}$.

$\mathbf{H}_{4}^{\prime}$ the operator $T: D(T) \rightarrow L^{2}\left(\mathbb{R}, \mathbb{R}^{m}\right)$ defined by

$$
D(T)=W_{\gamma}^{2,2}\left(\mathbb{R}, \mathbb{R}^{m}\right), \quad T v=-v^{\prime \prime}+W_{u u}(\bar{u}) v,
$$

where $W_{\gamma}^{2,2}\left(\mathbb{R}, \mathbb{R}^{m}\right) \subset W^{2,2}\left(\mathbb{R}, \mathbb{R}^{m}\right)$ is the subspace of the maps that satisfy $u(-s)=\gamma u(s)$, has a trivial kernel.

Then we have the assumptions concerning uniqueness and hyperbolicity of $\tilde{u}$ :

$\mathbf{H}_{5}$ There is a unique $T$-equivariant solution $\tilde{u}: \mathbb{R}^{2} \rightarrow \mathbb{R}^{m}$ of (1.3)

$$
\tilde{u}(g s)=g \tilde{u}(s), \text { for } g \in T \text { and } s \in \mathbb{R}^{2}
$$

that satisfies the estimate

$$
|\tilde{u}(s)-a| \leq K e^{-k d\left(s, \partial D_{2}\right)}, \text { for } s \in D_{2},
$$

where $D_{2}=\left\{s \in \mathbb{R}^{2}:\left|s_{2}\right|<\sqrt{3} s_{1}, s_{1}>0\right\}$.

$\mathbf{H}_{6}$ the operator $\mathcal{T}: D(\mathcal{T}) \rightarrow L^{2}\left(\mathbb{R}^{2}, \mathbb{R}^{m}\right)$ defined by

$$
D(\mathcal{T})=W_{T}^{2,2}\left(\mathbb{R}^{2}, \mathbb{R}^{m}\right), \quad \mathcal{T} v=-\Delta v+W_{u u}(\bar{u}) v,
$$

where $W_{T}^{2,2}\left(\mathbb{R}^{2}, \mathbb{R}^{m}\right) \subset W^{2,2}\left(\mathbb{R}^{2}, \mathbb{R}^{m}\right)$ is the subspace of $T$-equivariant maps, has a trivial kernel.

We are now in the position of stating:

Theorem 1.8. Assume that $W$ satisfies $\mathbf{H}_{1}$ and $\mathbf{H}_{2}$ with $a=(1,0, \ldots, 0)$ and moreover that $0=W(a)<W(u)$ for $u \in \bar{F} \backslash\{a\}$. Assume that $\mathbf{H}_{3}^{\prime}, \mathbf{H}_{4}^{\prime}$ and $\mathbf{H}_{5}, \mathbf{H}_{6}$ hold. Let $u: \mathbb{R}^{n} \rightarrow \mathbb{R}^{m}$, for $n \geq 3$ and $m \geq 2$, be a $T$-equivariant minimizer that satisfies (1.9) and, for some $\delta>0, d_{0} \geq 0$, the condition

$$
\left|u(x)-\gamma_{ \pm} a\right| \geq \delta \text { for } d(x, \partial D)>d_{0} \text { and } x \in D,
$$

where $D=\left\{x \in \mathbb{R}^{n}:\left|x_{2}\right|<\sqrt{3} x_{1}, x_{1}>0\right\}$.

Then $u$ is two-dimensional:

$$
u(x)=\tilde{u}\left(x_{1}, x_{2}\right), \text { for } x \in \mathbb{R}^{n} .
$$


Remark 1.9. If instead of a minimizer defined on $\mathbb{R}^{n}$ we considered a minimizer defined on a subset $\Omega \subset \mathbb{R}^{n}$, which in analogy with (1.7) satisfies

$x=\left(x_{1}, x_{2}, x_{3}, \ldots, x_{n}\right) \in \Omega$

$\Rightarrow\left(l \cos \phi, l \sin \phi, x_{3}, \ldots, x_{n}\right) \in \Omega$, for $l \in\left[0, \sqrt{x_{1}^{2}+x_{2}^{2}}\right]$ and $\phi \in[0,2 \pi)$,

instead of (1.25), the conclusion of Theorem 1.8 would be exponential convergence of $u$ to $\tilde{u}$ similar to $(1.15)$.

Theorem 1.8 is an example of a De Giorgi type result for systems where monotonicity is replaced by minimality (see $[2,12]$ and $[21$, Section 3$]$ ). In the symmetric setting we are considering, it is the PDE analog of the fact that a minimal cone $\mathcal{C}$ in $\mathbb{R}^{n}$ with the symmetry of the equilateral triangle is necessarily of the form $\mathcal{C}=\tilde{\mathcal{C}} \times \mathbb{R}^{n-2}$, with $\tilde{\mathcal{C}}$ the triod in the plane. The rest of the paper is devoted to the proofs. In Section 2 we prove Theorem 1.4. In Section 2.1 and Section 2.2 we prove a number of lemmas that are basic for the proof of Theorem 1.4 that we conclude in Sections 2.3 and 2.4. Theorems 1.7 and Theorem 1.8 are proved in Section 2.5 and Section 3.

\section{The proof of Theorem 1.4}

The proof of Theorem 1.4 that we present here, from an abstract point of view, has a lot in common with the proof of Theorem 1.2 in [15]. We will remark on this point later and spend a few words to motivate the various lemmas that compose the proof of Theorem 1.4. We begin with some notation and two basic lemmas.

If $E \subset \mathbb{R}^{d}$, for $d \geq 1$, is a measurable set we let $\mathcal{H}^{d}(E)$ be the $d$-dimensional Lebesgue measure of $\bar{E}$.

\subsection{Basic lemmas}

In the following we use the notation $x=(s, \xi)$ with $x_{1}=s$ and $\left(x_{2}, \ldots, x_{n}\right)=\xi$. From (1.11) it follows that, if $(l, \xi) \in \Omega^{+}$satisfies $d\left((l, \xi), \partial \Omega^{+}\right) \geq l$, then the map $s \rightarrow u(s, \xi), s \in[-l, l]$, that we still denote with $u$, satisfies the bound

$$
|u-a|+\left|u_{s}\right| \leq K_{0} e^{-k_{0} s}, \text { for } s \in[0, l] .
$$

Note that from the assumption that $a$ is nondegenerate in $\mathbf{H}_{2}$ we also have

$$
|\bar{u}-a|+\left|\bar{u}_{s}\right| \leq K_{0} e^{-k_{0} s}, \text { for } s \geq 0 .
$$

Let $T_{l}$ the operator defined by

$$
D_{l}\left(T_{l}\right)=\left\{v \in W_{S}^{2,2}\left([-l, l], \mathbb{R}^{m}\right): v( \pm l)=0\right\}, \quad T_{l} v=-v^{\prime \prime}+W_{u u}(\bar{u}) v .
$$

For $l \in(0,+\infty]$ we let $\langle v, w\rangle_{l}=\int_{-l}^{l} v w$ denote the inner product in $L^{2}\left((-l, l), \mathbb{R}^{m}\right)$.

We let $\|v\|_{l}=\langle v, v\rangle_{l}^{\frac{1}{2}}$ and $\|v\|_{1, l}=\|v\|_{W^{1,2}\left([-l, l], \mathbb{R}^{m}\right)}$.

For the standard inner product in $\mathbb{R}^{m}$ we use the notation $(\cdot, \cdot)$. 
If $v:[-l, l] \rightarrow \mathbb{R}^{m}$ satisfies (2.1), then $\|v\|_{1, l} \leq C=\frac{K_{0}}{\sqrt{k_{0}}}$. We set

$$
\mathcal{B}_{l}^{1,2}:=\left\{v \in W_{S}^{1,2}\left([-l, l], \mathbb{R}^{m}\right): v( \pm l)=0 ;\|v\|_{1, l} \leq C\right\},
$$

where $W_{S}^{1,2}\left([-l, l], \mathbb{R}^{m}\right)$ is the subspace of symmetric maps. Let $\mathbb{S}$ be defined by

$$
\mathbb{S}=\left\{v \in W_{S}^{1,2}\left([-l, l], \mathbb{R}^{m}\right): v( \pm l)=0 ;\|v\|_{l}=1\right\} .
$$

Lemma 2.1. Assume $\mathrm{H}_{1}$ and $\mathrm{H}_{2}$ as in Theorem 1.4 and let $\mathbf{e}_{l}: \mathcal{B}_{l}^{1,2} \rightarrow \mathbb{R}$ be defined by

$$
\mathbf{e}_{l}(v):=\frac{1}{2}\left(\left\langle\bar{u}_{s}+v_{s}, \bar{u}_{s}+v_{s}\right\rangle_{l}-\left\langle\bar{u}_{s}, \bar{u}_{s}\right\rangle_{l}\right)+\int_{-l}^{l}(W(\bar{u}+v)-W(\bar{u})) d s .
$$

Then there exist $l_{0}>0, q^{\circ}>0$ and $c>0$ such that, for all $l \geq l_{0}$, we have

$$
\begin{cases}D_{q q} \mathbf{e}_{l}(q v) \geq c^{2} & \text { for } q \in\left[0, q^{\circ}\right] \text { and } v \in \mathbb{S} \\ \mathbf{e}_{l}(q v) \geq \mathbf{e}_{l}\left(q^{\circ} v\right) & \text { for } q^{\circ} \leq q \text { and } v \in \mathbb{S}, \\ \mathbf{e}_{l}(q v) \geq \tilde{\mathbf{e}}_{l}(p, q, v) & \\ \quad:=\mathbf{e}_{l}(p v)+D_{q} \mathbf{e}_{l}(p v)(q-p) & \text { for } 0 \leq p<q \leq q^{\circ} \text { and } v \in \mathbb{S} \\ D_{p} \tilde{\mathbf{e}}_{l}(p, q, v) \geq 0 & \text { for } 0 \leq p<q \leq q^{\circ} \text { and } v \in \mathbb{S} .\end{cases}
$$

Remark 2.2. $\mathbf{e}_{l}$ is a kind of effective potential. Indeed, as we shall see, in the proof of Theorem 1.4 the map $L^{2}\left((-l, l), \mathbb{R}^{m}\right) \ni q \mapsto \mathbf{e}_{l}(q v)$ plays a role similar to the one of the usual potential $\mathbb{R} \ni q \mapsto W(a+q \nu)$ in the proof of [15, Theorem 1.2].

Proof. Differentiating twice $\mathbf{e}_{l}(q v)$ with respect to $q$ gives

$$
\begin{aligned}
D_{q q} \mathbf{e}_{l}(q v) & =\int_{-l}^{l}\left(v_{s}, v_{s}\right)+\int_{-l}^{l} W_{u u}(\bar{u}+q v)(v, v) \\
& =\left.D_{q q} \mathbf{e}_{l}(q v)\right|_{q=0}+\int_{-l}^{l}\left(W_{u u}(\bar{u}+q v)-W_{u u}(\bar{u})\right)(v, v) .
\end{aligned}
$$

By the interpolation inequality

$$
\|v\|_{L^{\infty}} \leq \sqrt{2}\|v\|_{1, l}^{\frac{1}{2}}\|v\|_{l}^{\frac{1}{2}} \leq \sqrt{2}\|v\|_{1, l}
$$

for $q v \in \mathcal{B}_{l}^{1,2}$ we get via the second inequality

$$
\|q v\|_{L^{\infty}} \leq \sqrt{2} C,
$$

and via the first

$$
\|v\|_{L^{\infty}} \leq \sqrt{2} C^{\frac{1}{2}} q^{-\frac{1}{2}} .
$$


Therefore we have

$$
\left|W_{u_{i} u_{j}}(\bar{u}(s)+q v(s))-W_{u_{i} u_{j}}(\bar{u}(s))\right| \leq \sqrt{2} C^{\frac{1}{2}} \bar{W}^{\prime \prime \prime} q^{\frac{1}{2}},
$$

where $\bar{W}^{\prime \prime \prime}$ is defined by

$$
\bar{W}^{\prime \prime \prime}:=\max _{\substack{1 \leq i, j, k \leq m \\ s \in \mathbb{R},|\tau| \leq 1, \tau \in \mathbb{R}^{m}}} W_{u_{i} u_{j} u_{k}}(\bar{u}(s)+\tau \sqrt{2} C) .
$$

From (2.12) we get

$$
\left|\int_{-l}^{l}\left(W_{u u}(\bar{u}+q v)-W_{u u}(\bar{u})\right)(v, v)\right| \leq C_{1} q^{\frac{1}{2}},
$$

where $C_{1}>0$ is a constant independent of $l$. We now observe that

$$
\left.D_{q q} \mathbf{e}_{l}(q v)\right|_{q=0}=\left\langle T_{l} v, v\right\rangle_{l}=\langle T \tilde{v}, \tilde{v}\rangle_{\infty},
$$

where $\tilde{v}$ is the trivial extension of $v$ to $\mathbb{R} . T$ is a self-adjoint operator which is positive by the minimality of $\bar{u}$. Therefore assumption $\mathbf{H}_{4}$ implies that the point spectrum of $T$ is bounded below by a positive number. From $\mathbf{H}_{2}$ the smallest eigenvalue $\mu$ of the matrix $W_{u u}(a)$ is positive and Persson's Theorem in [1] implies that also the remaining part of the spectrum of $T$, the essential spectrum, is bounded below by $\mu>0$. It follows that the spectrum of $T$ is bounded below by a positive constant $0<\tilde{\mu} \leq \mu$. From this (2.15) and Theorem 13.31 in [20] it follows

$$
\left.D_{q q} \mathbf{e}_{l}(q v)\right|_{q=0} \geq \tilde{\mu},
$$

which together with (2.14) implies

$$
D_{q q} \mathbf{e}_{l}(q v) \mid \geq \tilde{\mu} \geq c^{2}:=\frac{\tilde{\mu}}{2}, \quad \text { for } q \in[0, \bar{q}],
$$

where $\bar{q}=\frac{1}{4} \frac{\tilde{\mu}^{2}}{C_{1}}$. This concludes the proof of $(2.7)_{1}$. We now consider the problem

$$
\min _{\substack{v \in \mathcal{B}_{l}^{1,2} \\\|v\|_{l} \geq \bar{q}}} \mathbf{e}_{l}(v) .
$$

Since the constraint in problem (2.18) is closed with respect to weak convergence in $W_{0}^{1,2}$, if $\bar{v}_{l}$ is a minimizer of problem (2.18), we have $\bar{v}_{l} \neq 0$. This implies

$$
\mathbf{e}_{l}\left(\bar{v}_{l}\right)=\alpha_{l}>0
$$

Indeed the uniqueness assumption about $\bar{u}$ implies that $v \equiv 0$ is the unique minimizer of $\mathbf{e}_{l}$. We have

$$
\liminf _{l \rightarrow+\infty} \alpha_{l}=\alpha>0
$$


To prove this we assume that instead there is a sequence $l_{k}$ such that $\lim _{k \rightarrow+\infty} \alpha_{l_{k}}=$ 0 . We can also assume that the sequence $\tilde{\bar{v}}_{l_{k}}$ of the trivial extensions of $\bar{v}_{l_{k}}$ converges weakly in $W^{1,2}$ to a map $\bar{v}$ which by lower semicontinuity satisfies

$$
\mathbf{e}_{\infty}(\bar{v})=0
$$

This is in contradiction with the assumption that $v \equiv 0$ is the unique minimizer of $\mathbf{e}_{\infty}$. Indeed the constraint in problem (2.18) persists in the limit and implies $\bar{v} \neq 0$. This establishes (2.20) and concludes the proof of $(2.7)_{2}$ with $q^{\circ}=\min \left\{\bar{q}, \frac{\sqrt{2 \alpha}}{c}\right\}$. The last two inequalities in (2.7) are straightforward consequences of $(2.7)_{1}$.

Lemma 2.3. Let be $u$ as in Theorem 1.3 and assume that

$$
(l, \xi) \in \Omega^{+}, d((l, \xi), \partial \Omega) \geq l
$$

then there is a constant $C_{2}>0$ independent of $l>1$, such that

$$
\|u(\cdot, \xi)-\bar{u}\|_{L^{\infty}\left([-l, l], \mathbb{R}^{m}\right)} \leq C_{2}\|u(\cdot, \xi)-\bar{u}\|_{l}^{\frac{2}{3}} .
$$

Proof. From (2.22) $u(\cdot, \xi)$ satisfies (2.1). Therefore using also (2.2) there exists $\bar{s} \in[0, l]$ such that $|u(s, \xi)-\bar{u}(s)| \leq m=:|u(\bar{s}, \xi)-\bar{u}(\bar{s})|$. From this and $\left|u(\cdot, \xi)_{s}-\bar{u}_{s}\right| \leq 2 K_{0}$ it follows

$$
|u(s, \xi)-\bar{u}(s)| \geq m\left(1-2 K_{0}|s-\bar{s}|\right), \text { for } s \in[-l, l] \cap\left[\bar{s}-\frac{m}{2 K_{0}}, \bar{s}+\frac{m}{2 K_{0}}\right]
$$

and a simple computation gives (2.23).

Before continuing with the proof, we explain the meaning of the lemmas that follow. Given $l, r>0$ and $\varsigma \in \mathbb{R}^{n-1}$ we let $\mathcal{C}_{l}^{r}(\varsigma) \subset \mathbb{R}^{n}$ denote the cylinder

$$
\mathcal{C}_{l}^{r}(\varsigma):=\{(s, \xi):-l<s<l ;|\xi-\varsigma|<r\}
$$

Lemma 2.4, Lemma 2.5 and Lemma 2.6 describe successive deformations through which, for fixed $\lambda>0, \varrho>0$ and $\bar{q} \in\left(0, q^{\circ}\right)$, we transform the minimizer $u$ first into a map $v$ then into $w$ and finally into a map $w^{\bar{q}}$ that satisfies the conditions

$$
\begin{array}{ll}
w^{\bar{q}}=u, & \text { on } \Omega \backslash \mathcal{C}_{l+\lambda}^{r+2 \varrho}(\varsigma), \\
w^{\bar{q}}\left(l+\frac{\lambda}{2}, \xi\right)=\bar{u}\left(l+\frac{\lambda}{2}\right), & \text { for }|\xi-\varsigma| \leq r+\frac{\varrho}{2}, \\
\left\|w^{\bar{q}}(\cdot, \xi)-\bar{u}(\cdot)\right\|_{l+\frac{\lambda}{2}} \leq \bar{q}, & \text { for }|\xi-\varsigma| \leq r+\frac{\varrho}{2}
\end{array}
$$

The deformations described in these lemmas are complemented by precise quantitative estimates on the amount of energy required for the deformation (see (iii) in 
Lemma 2.4, (iii) in Lemma 2.5 and (2.47) in Lemma 2.6). Lemma 2.4 describes the deformation of $u$ into a map $v$ that coincides with $\bar{u}$ on the bases of $\mathcal{C}_{l+\frac{\lambda}{2}}^{r+\varrho}(\varsigma)$ :

$$
\begin{gathered}
v=u, \text { outside } \mathcal{C}_{l+\lambda}^{r+2 \varrho}(\varsigma) \backslash \overline{\mathcal{C}}_{l}^{r+2 \varrho}(\varsigma) \\
v\left(l+\frac{\lambda}{2}, \xi\right)=\bar{u}\left(l+\frac{\lambda}{2}\right), \text { for }|\xi-\varsigma| \leq r+\varrho .
\end{gathered}
$$

Lemma 2.5 describes the deformation of $v$ into a map $w$ that satisfies

$$
\begin{aligned}
w & =v, \text { outside } \mathcal{C}_{l+\frac{\lambda}{2}}^{r+\varrho}(\varsigma) \backslash \overline{\mathcal{C}}_{l+\frac{\lambda}{2}}^{r}(\varsigma) \\
\|w(\cdot, \xi)-\bar{u}(\cdot)\|_{l+\frac{\lambda}{2}} \leq \bar{q}, & \text { for }|\xi-\varsigma|=r+\frac{\varrho}{2}
\end{aligned}
$$

Lemma 2.6 describes the deformation of $w$ into $w^{\bar{q}}$. Lemma 2.7 and Corollary 2.8 show that we can replace $w^{\bar{q}}$ with a map $\omega$ that coincides with $w^{\bar{q}}$ outside $\mathcal{C}_{l+\frac{\lambda}{2}}^{r+\frac{Q}{2}}(\varsigma)$ and has less energy than $w^{\bar{q}}$. Moreover Corollary 2.8 yields a quantitative estimate for the energy difference.

In Section 2.3 we put all these energy estimates together and show (see Proposition 2.9) that, if $d\left(\mathcal{C}_{l+\lambda}^{r+2 \varrho}(\varsigma), \partial \Omega\right) \geq l+\lambda$ and $r, l$ are sufficiently large, the assumption

$$
\|u(\cdot, \varsigma)-\bar{u}(\cdot)\|_{l} \geq q^{\circ}
$$

is incompatible with the minimality of $u$, thus establishing the estimate

$$
\|u(\cdot, \varsigma)-\bar{u}(\cdot)\|_{l}<q^{\circ}
$$

which is the main step in the proof of Theorem 1.4.

\subsection{Replacement lemmas}

Lemma 2.4. Let $\lambda$ and $\varrho>0$ be fixed. Assume that $\mathcal{C}_{l+\lambda}^{r+2 \varrho}(\varsigma) \subset \Omega$ satisfies

$$
d\left(\mathcal{C}_{l+\lambda}^{r+2 \varrho}(\varsigma), \partial \Omega\right) \geq l+\lambda
$$

Then there exists a map $v \in C_{S}^{\operatorname{Exp}}\left(\bar{\Omega}, \mathbb{R}^{m}\right)$ such that

(i) $v=u$, on $\bar{\Omega} \backslash\left(\mathcal{C}_{l+\lambda}^{r+2 \varrho}(\varsigma) \backslash \overline{\mathcal{C}}_{l}^{r+2 \varrho}(\varsigma)\right)$,

(ii) $v\left(l+\frac{\lambda}{2}, \xi\right)=\bar{u}\left(l+\frac{\lambda}{2}\right)$, for $|\xi-\varsigma| \leq r+\varrho$.

(iii) $J_{\mathcal{C}_{l+\lambda}^{r+2 \varrho}(\varsigma)}(v)-J_{\mathcal{C}_{l+\lambda}^{r+2 \varrho}(\varsigma)}(u) \leq C_{0} r^{n-1} e^{-2 k_{0} l}$,

where $C_{0}>0$ is a constant independent of $l$ and $r$. 
Proof. For $(s, \xi) \in \overline{\mathcal{C}}_{l+\lambda}^{r+\varrho}(\varsigma) \backslash \mathcal{C}_{l}^{r+\varrho}(\varsigma)$ we define $v$ by

$$
\begin{aligned}
& v(s, \xi)=\left(1-\left|1-2 \frac{s-l}{\lambda}\right|\right) \bar{u}(s)+\left|1-2 \frac{s-l}{\lambda}\right| u(s, \xi), \\
& \qquad \text { for } s \in[l, l+\lambda] \text { and }|\xi-\varsigma| \leq r+\varrho .
\end{aligned}
$$

It remains to define $v(s, \xi)$ for $(s, \xi) \in(l, l+\lambda) \times\{\xi: r+\varrho<|\xi-\varsigma|<r+2 \varrho\}$

Set

$$
\begin{aligned}
B u(s, \xi) & =\frac{l+\lambda-s}{\lambda} u(l, \xi)+\frac{s-l}{\lambda} u(l+\lambda, \xi), \\
\tilde{u}(s, \xi) & =u(s, \xi)-B u(s, \xi) .
\end{aligned}
$$

Note that by (2.30) $|\xi-\varsigma|=r+\varrho$ implies $v(l, \xi)=u(l, \xi), v(l+\lambda, \xi)=$ $u(l+\lambda, \xi)$ and therefore we have

$$
|\xi-\varsigma|=r+\varrho \Rightarrow B u(s, \xi)=B v(s, \xi), \text { for } s \in[l, l+\lambda]
$$

where $v$ is defined in (2.30). Set, for $s \in[l, l+\lambda]$

$$
\hat{v}(s, \xi)=v\left(s,(r+\varrho) \frac{\xi-\varsigma}{|\xi-\varsigma|}+\varsigma\right)-B u\left(s,(r+\varrho) \frac{\xi-\varsigma}{|\xi-\varsigma|}+\varsigma\right),
$$

where again $v$ is defined in (2.30). With these notations we complete the definition of $v$ by setting

$$
\begin{array}{r}
v(s, \xi)=B u(s, \xi)+\frac{|\xi-\varsigma|-r-\varrho}{\varrho} \tilde{u}(s, \xi)+\frac{2 \varrho+r-|\xi-\varsigma|}{\varrho} \hat{v}(s, \xi), \\
\quad \text { for }(s, \xi) \in(l, l+\lambda) \times\{\xi: r+\varrho<|\xi-\varsigma|<r+2 \varrho\} .
\end{array}
$$

Statements (i) and (ii) are obvious consequences of the definition of $v$. Direct inspection of (2.30) and (2.34) shows that $v$ is continuous. From $(2.30) v(s, \xi)$ is a linear combination of $\bar{u}(s)$ and $u(s, \xi)$ computed for $s \in[l, l+\lambda]$. A similar statement applies to $v(s, \xi)$ in (2.34) since $B u(s, \xi), \hat{v}(s, \xi)$ and $\tilde{u}(s, \xi)$ are linear combinations of $u(s, \xi)$ and $v(s, \xi)$ in (2.30) computed for $s \in[l, l+\lambda]$. From this, assumption (2.29) and (2.1) we conclude

$$
|v-a|+|\nabla v| \leq C_{3} e^{-k_{0} l} \text { for }(s, \xi) \in \mathcal{C}_{l+\lambda}^{r+2 \varrho}(\varsigma) \backslash \overline{\mathcal{C}}_{l}^{r+2 \varrho}(\varsigma),
$$

where $C_{3}>0$ is a constant independent of $l$ and $r$. From (2.35) and the assumptions on the potential $W$ it follows, for $(s, \xi) \in \mathcal{C}_{l+\lambda}^{r+2 \varrho}(\varsigma) \backslash \overline{\mathcal{C}}_{l}^{r+2 \varrho}(\varsigma)$,

$$
\frac{1}{2}|\nabla v|^{2}+W(v) \leq C_{4} e^{-2 k_{0} l}
$$

which together with $\mathcal{H}^{n}\left(\mathcal{C}_{l+\lambda}^{r+2 \varrho}(\varsigma) \backslash \overline{\mathcal{C}}_{l}^{r+2 \varrho}(\varsigma)\right) \leq C_{5} r^{n-1}$ concludes the proof. 
Given a number $0<\bar{q}<q^{\circ}$, let $A_{\bar{q}} \subset \mathbb{R}^{n-1}$ be the set

$$
A_{\bar{q}}:=\left\{\xi:\|v(\cdot, \xi)-\bar{u}(\cdot)\|_{l+\frac{\lambda}{2}}>\bar{q},|\xi-\varsigma|<r+\varrho\right\}
$$

where $v$ is the map constructed in Lemma 2.4.

Lemma 2.5. Let $v$ as before and let $S:=A_{\bar{q}} \cap\{\xi: r<|\xi-\varsigma|<r+\varrho\}$. Then there is a constant $C_{1}>0$ independent of $l$ and $r$ and a map $w \in C_{S}^{\operatorname{Exp}}\left(\bar{\Omega}, \mathbb{R}^{m}\right)$ such that

(i) $w=v$ on $\bar{\Omega} \backslash\left(\mathcal{C}_{l+\frac{\lambda}{2}}^{r+\varrho}(\varsigma) \backslash \overline{\mathcal{C}}_{l+\frac{\lambda}{2}}^{r}(\varsigma)\right)$

(ii) $\|w(\cdot, \xi)-\bar{u}(\cdot)\|_{l+\frac{\lambda}{2}} \leq \bar{q}$, for $|\xi-\varsigma|=r+\frac{\varrho}{2}$.

(iii) $J_{\mathcal{C}_{l+\frac{\lambda}{2}}^{r+\varrho}(\varsigma) \backslash \overline{\mathcal{C}}_{l+\frac{\lambda}{2}}^{r}(\varsigma)}(w)-J_{\mathcal{C}_{l+\frac{\lambda}{2}}^{r+\varrho}(\varsigma) \backslash \overline{\mathcal{C}}_{l+\frac{\lambda}{2}}^{r}(\varsigma)}(v) \leq C_{1} \mathcal{H}^{n-1}(S)$.

Proof. Set

$$
\begin{aligned}
& q^{v}(\xi)=\|v(\cdot, \xi)-\bar{u}(\cdot)\|_{l+\frac{\lambda}{2}}, \\
& v^{v}(s, \xi)=\frac{v(s, \xi)-\bar{u}(s)}{q^{v}(\xi)}, \quad \text { for } s \in\left(-l-\frac{\lambda}{2}, l+\frac{\lambda}{2}\right), \text { and } \xi \in S .
\end{aligned}
$$

For $s \in\left(-l-\frac{\lambda}{2}, l+\frac{\lambda}{2}\right)$, and $\xi \in S$, define

$$
\begin{aligned}
& w(s, \xi)=\bar{u}(s)+q^{w}(\xi) v^{v}(s, \xi) \\
& q^{w}(\xi)=\left(1-\left|1-2 \frac{|\xi-\varsigma|-r}{\varrho}\right|\right) \bar{q}+\left|1-2 \frac{|\xi-\varsigma|-r}{\varrho}\right| q^{v}(\xi) .
\end{aligned}
$$

From this definition it follows that $w$ coincides with $v=\bar{u}+q^{v} v^{v}$ if $|\xi-\varsigma|=r$ or $|\xi-\varsigma|=r+\varrho$ or $q^{v}=\bar{q}$. This shows that $w$ coincides with $v$ on the boundary of the set $\left(-l-\frac{\lambda}{2}, l+\frac{\lambda}{2}\right) \times S$ and implies (i). From (2.39) it also follows that $q^{w}=\bar{q}$ for $|\xi-\varsigma|=r+\frac{\varrho}{2}$ for $\xi \in S$. This and the definition of $S$ imply (ii). To prove (iii) we note that

$$
|w-\bar{u}|=\left|q^{w} v^{v}\right| \leq\left|q^{v} v^{v}\right|=|v-\bar{u}|, \text { for } s \in\left(-l-\frac{\lambda}{2}, l+\frac{\lambda}{2}\right), \text { and } \xi \in S
$$

which implies

$$
|w-a| \leq K_{0} e^{-k_{0} s}, \text { for } s \in\left(0, l+\frac{\lambda}{2}\right), \text { and } \xi \in S .
$$

Therefore we have

$$
\int_{-l-\frac{\lambda}{2}}^{l+\frac{\lambda}{2}}(W(w)-W(v)) \leq \int_{-l-\frac{\lambda}{2}}^{l+\frac{\lambda}{2}} W(w) \leq C, \text { for } \xi \in S .
$$


We can write

$$
w=\frac{q^{w}}{q^{v}}(v-\bar{u}), \text { for } s \in\left(0, l+\frac{\lambda}{2}\right), \text { and } \xi \in S
$$

therefore we have, using also $v \in C_{S}^{\operatorname{Exp}}\left(\bar{\Omega}, \mathbb{R}^{m}\right)$ and (2.2)

$$
\begin{aligned}
& w_{s}=\frac{q^{w}}{q^{v}}\left(v_{s}-\bar{u}_{s}\right) \Rightarrow\left|w_{s}\right| \leq K e^{-k|s|}, \\
& w_{\xi_{j}}=\left(\frac{q^{w}}{q^{v}}\right)_{\xi_{j}}(v-\bar{u})+\frac{q^{w}}{q^{v}} v_{\xi_{j}} .
\end{aligned}
$$

From $q_{\xi_{j}}^{v}=\left\langle v^{v}, v_{\xi_{j}}\right\rangle_{l+\frac{\lambda}{2}}$ and (2.39) it follows

$$
\begin{aligned}
\left(\frac{q^{w}}{q^{v}}\right)_{\xi_{j}}= & \left|1-2 \frac{|\xi-\varsigma|-r}{\varrho}\right|_{\xi_{j}}\left(1-\frac{\bar{q}}{q^{v}}\right) \\
& -\left(1-\left|1-2 \frac{|\xi-\varsigma|-r}{\varrho}\right|\right) \frac{\bar{q}}{\left(q^{v}\right)^{2}}\left\langle v^{v}, v_{\xi_{j}}\right\rangle_{l+\frac{\lambda}{2}}, \\
\Rightarrow & \left|\left(\frac{q^{w}}{q^{v}}\right)_{\xi_{j}}\right| \leq \frac{2}{\varrho}+\frac{1}{q^{v}}\left\|v_{\xi_{j}}\right\|_{l+\frac{\lambda}{2}},
\end{aligned}
$$

where we have used $\frac{\bar{q}}{q^{v}} \leq 1$ for $\xi \in S$. From (2.43) and (2.44) it follows $\left|w_{\xi_{j}}\right| \leq\left(\frac{2}{\varrho}+\frac{\left\|v_{\xi_{j}}\right\|_{l+\frac{\lambda}{2}}}{\bar{q}}\right)|v-\bar{u}|+\left|v_{\xi_{j}}\right| \leq K e^{-k_{0}|s|}$, for $s \in\left(-l-\frac{\lambda}{2}, l+\frac{\lambda}{2}\right)$, and $\xi \in S$, where again we have used $v \in C_{S}^{\operatorname{Exp}}\left(\bar{\Omega}, \mathbb{R}^{m}\right)$ and (2.2). From this and (2.43) we conclude

$$
\int_{-l-\frac{\lambda}{2}}^{l+\frac{\lambda}{2}}\left(|\nabla w|^{2}-|\nabla v|^{2}\right) \leq \int_{-l-\frac{\lambda}{2}}^{l+\frac{\lambda}{2}}|\nabla w|^{2} \leq C, \text { for } \xi \in S .
$$

This inequality and (2.42) conclude the proof.

Lemma 2.6. Let $w$ the map constructed in Lemma 2.5. Define $w^{\bar{q}}$ by setting

$$
w^{\bar{q}}= \begin{cases}\bar{u}+\bar{q} \nu^{v} \text { for }(s, \xi) \in \mathcal{C}_{l+\frac{\lambda}{2}}^{r+\frac{\rho}{2}}(\varsigma), \text { and } \xi \in A_{\bar{q}} \\ w \quad \text { for }(s, \xi) \in \mathcal{C}_{l+\frac{\lambda}{2}}^{r+\frac{\rho}{2}}(\varsigma), \xi \notin A_{\bar{q}}, \text { and }(s, \xi) \notin \mathcal{C}_{l+\frac{\lambda}{2}}^{r+\frac{\rho}{2}}(\varsigma) .\end{cases}
$$

Then $w^{\bar{q}} \in C_{S}^{\operatorname{Exp}}\left(\bar{\Omega}, \mathbb{R}^{m}\right)$ and

$$
J_{\mathcal{C}_{l+\frac{\lambda}{2}}^{r+\frac{\rho}{2}}(\varsigma)}\left(w^{\bar{q}}\right)-J_{\mathcal{C}_{l+\frac{\lambda}{2}}^{r+\frac{\rho}{2}}(\varsigma)}(w) \leq 0
$$


Proof. We have $w-\bar{u}=q^{w} v^{w}$ and $q^{w}>\bar{q}$ on $A_{\bar{q}}$. Thus, recalling the definition of $\mathbf{e}_{l}$, Lemma 2.1 and $\bar{q} \leq q^{\circ}$, we have $\mathbf{e}_{l+\frac{\lambda}{2}}\left(\bar{q} v^{w}\right)-\mathbf{e}_{l+\frac{\lambda}{2}}\left(q^{w} v^{w}\right) \leq 0$ and therefore

$$
\begin{aligned}
J_{\mathcal{C}_{l+\frac{\lambda}{2}}^{r+\frac{\rho}{2}}(\varsigma)}\left(w^{\bar{q}}\right)-J_{\mathcal{C}_{l+\frac{\lambda}{2}}(\varsigma)}(w)= & \int_{\tilde{A}_{\bar{q}}}\left(\mathbf{e}_{l+\frac{\lambda}{2}}\left(\bar{q} v^{w}\right)-\mathbf{e}_{l+\frac{\lambda}{2}}\left(q^{w} v^{w}\right)\right) d \xi \\
& +\frac{1}{2} \sum_{j} \int_{\tilde{A}_{\bar{q}}}\left(\left\langle w_{\xi_{j}}^{\bar{q}}, w_{\xi_{j}}^{\bar{q}}\right\rangle_{l+\frac{\lambda}{2}}-\left\langle w_{\xi_{j}}, w_{\xi_{j}}\right\rangle_{l+\frac{\lambda}{2}}\right) d \xi \\
\leq & \frac{1}{2} \sum_{j} \int_{\tilde{A}_{\bar{q}}}\left(\left\langle w_{\xi_{j}}^{\bar{q}}, w_{\xi_{j}}^{\bar{q}}\right\rangle_{l+\frac{\lambda}{2}}-\left\langle w_{\xi_{j}}, w_{\xi_{j}}\right\rangle_{l+\frac{\lambda}{2}}\right) d \xi
\end{aligned}
$$

where we have set $\tilde{A}_{\bar{q}}=A_{\bar{q}} \cap B_{r+\frac{\varrho}{2}}(\varsigma)$ with $B_{r+\frac{\varrho}{2}}(\varsigma)=\left\{|\xi-\varsigma| \leq r+\frac{\varrho}{2}\right\}$.

To conclude the proof we note that for $\xi \in \tilde{A}_{\bar{q}}$

$$
\begin{aligned}
& w_{\xi_{j}}^{\bar{q}}=\bar{q} v_{\xi_{j}}^{v}, \Rightarrow\left\langle w_{\xi_{j}}^{\bar{q}}, w_{\xi_{j}}^{\bar{q}}\right\rangle_{l+\frac{\lambda}{2}}=\bar{q}^{2}\left\langle v_{\xi_{j}}^{v}, v_{\xi_{j}}^{v}\right\rangle_{l+\frac{\lambda}{2}}, \\
& w_{\xi_{j}}=q_{\xi_{j}}^{w} v+q^{w} v_{\xi_{j}}^{v}, \Rightarrow\left\langle w_{\xi_{j}}, w_{\xi_{j}}\right\rangle_{l+\frac{\lambda}{2}}=\left(q_{\xi_{j}}^{w}\right)^{2}+\left(q^{w}\right)^{2}\left\langle v_{\xi_{j}}^{v}, v_{\xi_{j}}^{v}\right\rangle_{l+\frac{\lambda}{2}},
\end{aligned}
$$

where we have also used the fact that $\left\langle v^{v}, v^{v}\right\rangle_{l+\frac{\lambda}{2}}=1$ implies $\left\langle v^{v}, v_{\xi_{j}}^{v}\right\rangle_{l+\frac{\lambda}{2}}=0$. From (2.49) it follows

$$
\left\langle w_{\xi_{j}}^{\bar{q}}, w_{\xi_{j}}^{\bar{q}}\right\rangle_{l+\frac{\lambda}{2}}-\left\langle w_{\xi_{j}}, w_{\xi_{j}}\right\rangle_{l+\frac{\lambda}{2}}=-\left(q_{\xi_{j}}^{v}\right)^{2}+\left(\bar{q}^{2}-\left(q^{w}\right)^{2}\right)\left\langle v_{\xi_{j}}^{v}, v_{\xi_{j}}^{v}\right\rangle_{l+\frac{\lambda}{2}} \leq 0,
$$

for $\xi \in \tilde{A}_{\bar{q}}$. This and (2.48) prove (2.47).

Next we show that we can associate to $w^{\bar{q}}$ a map $\omega \in C_{S}^{\operatorname{Exp}}\left(\bar{\Omega}, \mathbb{R}^{m}\right)$ which coincides with $w^{\bar{q}}$ on $\Omega \backslash \mathcal{C}_{l+\frac{\lambda}{2}}^{r+\frac{\varrho}{2}}(\varsigma)$ and has less energy than $w^{\bar{q}}$. Moreover we derive a quantitative estimate of the energy difference. We follow closely the argument in [15]. First we observe that, if we define $q^{*}(\xi):=q^{w^{\bar{q}}}(\xi)$, we can represent $J_{\mathcal{C}_{l+\frac{\lambda}{2}}^{r+\frac{\rho}{2}}(\varsigma)}\left(w^{\bar{q}}\right)$ in the polar form

$$
\begin{aligned}
& J_{\mathcal{C}_{l+\frac{\lambda}{2}}^{r+\frac{\rho}{2}}(\varsigma)}\left(w^{\bar{q}}\right)-J_{\mathcal{C}_{l+\frac{\lambda}{2}}^{r+\frac{\rho}{2}}(\varsigma)}(\bar{u}) \\
& =\int_{B_{r+\frac{\varrho}{2}}(\varsigma) \cap\left\{q^{*}>0\right\}}\left(\frac{1}{2}\left(\left|\nabla q^{*}\right|^{2}+q^{* 2} \sum_{j}\left\langle v_{\xi_{j}}^{w}, v_{\xi_{j}}^{w}\right\rangle_{l+\frac{\lambda}{2}}\right)+\mathbf{e}_{l+\frac{\lambda}{2}}\left(q^{*} v^{w}\right)\right) d \xi .
\end{aligned}
$$

This follows from $v^{w}=v^{v}$ and from $\left\langle v^{v}, v_{\xi_{j}}^{v}\right\rangle_{l+\frac{\lambda}{2}}=0$, which imply

$$
\sum_{j}\left\langle w_{\xi_{j}}^{\bar{q}}, w_{\xi_{j}}^{\bar{q}}\right\rangle_{l+\frac{\lambda}{2}}=\left|\nabla q^{*}\right|^{2}+q^{* 2} \sum_{j}\left\langle v_{\xi_{j}}^{w}, v_{\xi_{j}}^{w}\right\rangle_{l+\frac{\lambda}{2}}
$$


and from the definition of $\mathbf{e}_{l}$ in Lemma 2.1. We remark that the definitions of $q^{*}$ and $w^{\bar{q}}$ imply

$$
\begin{aligned}
& q^{*} \leq \bar{q}, \text { on } B_{r+\frac{\varrho}{2}}(\varsigma), \\
& q^{*}=\bar{q}, \text { on } A_{\bar{q}} \cap B_{r+\frac{\varrho}{2}}(\varsigma) .
\end{aligned}
$$

Lemma 2.7. Let $c>0$ as in Lemma 2.1 and let $\varphi: B_{r+\frac{\varrho}{2}}(\varsigma) \subset \mathbb{R}^{n-1} \rightarrow \mathbb{R}$ be the solution of

$$
\begin{cases}\Delta \varphi=c^{2} \varphi & \text { in } B_{r+\frac{\varrho}{2}}(\varsigma) \\ \varphi=\bar{q} & \text { on } \partial B_{r+\frac{\rho}{2}}(\varsigma)\end{cases}
$$

Then there is a map $\omega \in C_{S}^{\operatorname{Exp}}\left(\bar{\Omega}, \mathbb{R}^{m}\right)$ with the following properties

$$
\begin{cases}\omega=w^{\bar{q}} & \text { on } \Omega \backslash \mathcal{C}_{l+\frac{\lambda}{2}}^{r+\frac{\varrho}{2}}(\varsigma) \\ \omega=q^{\omega} v^{w}+\bar{u} & \text { on } \mathcal{C}_{l+\frac{\lambda}{2}}^{r+\frac{\varrho}{2}}(\varsigma) \\ q^{\omega} \leq \varphi \leq \bar{q} & \text { on } B_{r+\frac{\varrho}{2}}(\varsigma)\end{cases}
$$

Moreover

$$
\begin{aligned}
& J_{\mathcal{C}_{l+\frac{\lambda}{2}}^{r+\frac{\varrho}{2}}(\varsigma)}\left(w^{\bar{q}}\right)-J_{\mathcal{C}_{l+\frac{\lambda}{2}}^{r+\frac{\varrho}{2}}(\varsigma)}(\omega) \\
& \geq \int_{B_{r+\frac{\varrho}{2}}(\varsigma) \cap\left\{q^{*}>\varphi\right\}}\left(\mathbf{e}_{l+\frac{\lambda}{2}}\left(q^{*} \nu^{w}\right)-\mathbf{e}_{l+\frac{\lambda}{2}}\left(\varphi v^{w}\right)-D_{q} \mathbf{e}_{l+\frac{\lambda}{2}}\left(\varphi v^{w}\right)\left(q^{*}-\varphi\right)\right) d \xi .
\end{aligned}
$$

Proof. Let $b>0, b \leq \min _{\xi \in B_{r+\frac{\rho}{2}}}(\varsigma) \varphi$ be fixed and let $A_{b} \subset B_{r+\frac{\rho}{2}}(\varsigma)$ the set $A_{b}:=\left\{\xi \in B_{r+\frac{\varrho}{2}}(\varsigma): q^{*}>b\right\} . A_{b}$ is an open set since $w^{\bar{q}}=\bar{u}+q^{*} v^{w}$ is continuous by construction. Let

$$
\mathcal{J}_{A_{b}}(p)=\int_{A_{b}}\left(\frac{1}{2}|\nabla p|^{2}+\mathbf{e}_{l+\frac{\lambda}{2}}\left(|p| v^{w}\right)\right) d \xi
$$

Since $A_{b}$ is open and $q^{*} \in L^{\infty}\left(A_{b}, \mathbb{R}\right)$ there exists a minimizer $p^{*} \in q^{*}+$ $W_{0}^{1,2}\left(A_{b}, \mathbb{R}\right)$ of the problem

$$
\mathcal{J}_{A_{b}}\left(p^{*}\right)=\min _{q^{*}+W_{0}^{1,2}\left(A_{b}, \mathbb{R}\right)} \mathcal{J}_{A_{b}}(p)
$$

We also have

$$
0 \leq p^{*} \leq \bar{q}
$$


This follows from (2.7) that implies $\mathcal{J}_{A_{b}}\left(\frac{p^{*}+\left|p^{*}\right|}{2}\right) \leq \mathcal{J}_{A_{b}}\left(p^{*}\right)$ and therefore $p^{*} \geq 0$. The other inequality is a consequence of $\mathcal{J}_{A_{b}}\left(\min \left\{p^{*}, \bar{q}\right\}\right) \leq \mathcal{J}_{A_{b}}\left(p^{*}\right)$ which follows from

$$
\int_{A_{b}}\left|\nabla\left(\min \left\{p^{*}, \bar{q}\right\}\right)\right|^{2} \leq \int_{A_{b}}\left|\nabla p^{*}\right|^{2}
$$

and from (2.7). Since the map $\left.q \rightarrow \mathbf{e}_{l+\frac{\lambda}{2}}\left(|q| v^{w}\right)\right)$ is a $C^{1}$ map, we can write the variational equation

$$
\int_{A_{b}}\left(\left(\nabla p^{*}, \nabla \gamma\right)+D_{q} \mathbf{e}_{l+\frac{\lambda}{2}}\left(p^{*} v^{w}\right) \gamma\right) d \xi=0
$$

for all $\gamma \in W_{0}^{1,2}\left(A_{b}, \mathbb{R}\right) \cap L^{\infty}\left(A_{b}\right)$. In particular, if we define $A_{b}^{*}:=\left\{x \in A_{b}\right.$ : $\left.p^{*}>\varphi\right\}$, we have

$$
\int_{A_{b}^{*}}\left(\left(\nabla p^{*}, \nabla \gamma\right)+D_{q} \mathbf{e}_{l+\frac{\lambda}{2}}\left(p^{*} v^{w}\right) \gamma\right) d \xi=0,
$$

for all $\gamma \in W_{0}^{1,2}\left(A_{b}, \mathbb{R}\right) \cap L^{\infty}\left(A_{b}\right)$ that vanish on $A_{b} \backslash A_{b}^{*}$. If we take $\gamma=\left(p^{*}-\varphi\right)^{+}$ in (2.59) and use (2.7) which implies $D_{q} \mathbf{e}_{l+\frac{\lambda}{2}}\left(p^{*} v^{w}\right) \geq c^{2} p^{*}$ we get

$$
\int_{A_{b}^{*}}\left(\left(\nabla p^{*}, \nabla\left(p^{*}-\varphi\right)\right)+c^{2} p^{*}\left(p^{*}-\varphi\right)\right) d \xi \leq 0 .
$$

This inequality and

$$
\int_{A_{b}^{*}}\left(\left(\nabla \varphi, \nabla\left(p^{*}-\varphi\right)\right)+c^{2} \varphi\left(p^{*}-\varphi\right)\right) d \xi=0,
$$

that follows from (2.52) imply

$$
\int_{A_{b}^{*}}\left(\left|\nabla\left(p^{*}-\varphi\right)\right|^{2}+c^{2}\left(p^{*}-\varphi\right)^{2}\right) d \xi \leq 0 .
$$

That is $\mathcal{H}^{n}\left(A_{b}^{*}\right)=0$ which together with $p^{*} \leq \varphi$ on $A_{b} \backslash A_{b}^{*}$ shows that

$$
p^{*} \leq \varphi, \text { for } \xi \in A_{b}
$$

Let $\omega$ be the map defined by setting

$$
\omega= \begin{cases}w^{\bar{q}} & \text { for }(s, \xi) \in \Omega \backslash\left(-l-\frac{\lambda}{2}, l+\frac{\lambda}{2}\right) \times A_{b} \\ \bar{u}+q^{\omega} v^{w}=\bar{u}+\min \left\{p^{*}, q^{*}\right\} v^{w} & \text { for } \xi \in A_{b} .\end{cases}
$$


Note that this definition, the definition of $A_{b}$ and (2.63) imply

$$
q^{\omega} \leq \varphi, \text { for } \xi \in B_{r+\frac{\varrho}{2}}(\varsigma) .
$$

From (2.64) we have

$$
\begin{aligned}
& J_{\mathcal{C}_{l+\frac{\lambda}{2}}^{r+\frac{\varrho}{2}}(\varsigma)}\left(w^{\bar{q}}\right)-J_{\mathcal{C}_{l+\frac{\lambda}{2}}^{r+\frac{\varrho}{2}}(\varsigma)}(\omega) \\
& \geq \int_{A_{b} \cap\left\{p^{*}<q^{*}\right\}}\left(\frac{1}{2}\left(\left|\nabla q^{*}\right|^{2}-\left|\nabla p^{*}\right|^{2}+\left(\left(q^{*}\right)^{2}-\left(p^{*}\right)^{2}\right) \sum_{j=1}^{n}\left\langle v_{\xi_{j}}^{w}, v_{\xi_{j}}^{w}\right\rangle_{l+\frac{\lambda}{2}}\right)\right. \\
& \left.\quad+\mathbf{e}_{l+\frac{\lambda}{2}}\left(q^{*} v^{w}\right)-\mathbf{e}_{l+\frac{\lambda}{2}}\left(p^{*} v^{w}\right)\right) d \xi \\
& \geq \int_{A_{b} \cap\left\{p^{*}<q^{*}\right\}}\left(\frac{1}{2}\left|\nabla q^{*}\right|^{2}-\left|\nabla p^{*}\right|^{2}+\mathbf{e}_{l+\frac{\lambda}{2}}\left(q^{*} v^{w}\right)-\mathbf{e}_{l+\frac{\lambda}{2}}\left(p^{*} v^{w}\right)\right) d \xi \\
& \geq \int_{A_{b} \cap\left\{p^{*}<q^{*}\right\}}\left(\frac{1}{2}\left|\nabla q^{*}-\nabla p^{*}\right|^{2}\right. \\
& \left.\quad+\mathbf{e}_{l+\frac{\lambda}{2}}\left(q^{*} v^{w}\right)-\mathbf{e}_{l+\frac{\lambda}{2}}\left(p^{*} v^{w}\right)-D_{q} \mathbf{e}_{l+\frac{\lambda}{2}}\left(p^{*} v^{w}\right)\left(q^{*}-p^{*}\right)\right) d \xi \geq 0 .
\end{aligned}
$$

where we have used

$$
\frac{1}{2}\left(\left|\nabla q^{*}\right|^{2}-\left|\nabla p^{*}\right|^{2}\right)=\frac{1}{2}\left|\nabla q^{*}-\nabla p^{*}\right|^{2}+\left(\nabla p^{*}, \nabla\left(q^{*}-p^{*}\right)\right)
$$

and

$$
\int_{A_{b} \cap\left\{p^{*}<q^{*}\right\}}\left(\nabla p^{*}, \nabla\left(q^{*}-p^{*}\right)\right) d \xi=-\int_{A_{b} \cap\left\{p^{*}<q^{*}\right\}} D_{q} \mathbf{e}_{l+\frac{\lambda}{2}}\left(p^{*} v^{w}\right)\left(q^{*}-p^{*}\right) d \xi
$$

which follows from (2.58) with $\gamma=\left(q^{*}-p^{*}\right)^{+}$. From $(2.7)_{3}$ and (2.63) we have

$$
\mathbf{e}_{l+\frac{\lambda}{2}}\left(q^{*} v^{w}\right)-\tilde{\mathbf{e}}_{l+\frac{\lambda}{2}}\left(p^{*}, q^{*}, v^{w}\right) \geq \mathbf{e}_{l+\frac{\lambda}{2}}\left(q^{*} v^{w}\right)-\tilde{\mathbf{e}}_{l+\frac{\lambda}{2}}\left(\varphi, q^{*}, v^{w}\right) .
$$

From this and (2.65) which implies

$$
B_{r+\frac{\varrho}{2}}(\varsigma) \cap\left\{\phi<q^{*}\right\}=A_{b} \cap\left\{\phi<q^{*}\right\} \subset A_{b} \cap\left\{p^{*}<q^{*}\right\},
$$

we have

$$
\begin{aligned}
& \int_{A_{b} \cap\left\{p^{*}<q^{*}\right\}}\left(\mathbf{e}_{l+\frac{\lambda}{2}}\left(q^{*} v^{w}\right)-\mathbf{e}_{l+\frac{\lambda}{2}}\left(p^{*} v^{w}\right)-D_{q} \mathbf{e}_{l+\frac{\lambda}{2}}\left(p^{*} v^{w}\right)\left(q^{*}-p^{*}\right)\right) d \xi \\
\geq & \int_{B_{r+\frac{\rho}{2}}(\varsigma) \cap\left\{\varphi<q^{*}\right\}}\left(\mathbf{e}_{l+\frac{\lambda}{2}}\left(q^{*} v^{w}\right)-\mathbf{e}_{l+\frac{\lambda}{2}}\left(p^{*} v^{w}\right)-D_{q} \mathbf{e}_{l+\frac{\lambda}{2}}\left(p^{*} v^{w}\right)\left(q^{*}-p^{*}\right)\right) d \xi \\
\geq & \int_{B_{r+\frac{\rho}{2}}(\varsigma) \cap\left\{\varphi<q^{*}\right\}}\left(\mathbf{e}_{l+\frac{\lambda}{2}}\left(q^{*} v^{w}\right)-\mathbf{e}_{l+\frac{\lambda}{2}}\left(\varphi v^{w}\right)-D_{q} \mathbf{e}_{l+\frac{\lambda}{2}}\left(\varphi v^{w}\right)\left(q^{*}-\varphi\right)\right) d \xi .
\end{aligned}
$$

The inequality (2.54) follows from this and (2.66). 
Corollary 2.8. Let $w^{\bar{q}}$ be as before and let $\omega \in C_{S}^{\operatorname{Exp}}\left(\bar{\Omega}, \mathbb{R}^{m}\right)$ be the map constructed in Lemma 2.7. Then there are $l_{0}>0, r_{0}>0$ and a number $c_{1}>0$ independent of $l \geq l_{0}, r \geq r_{0}$ such that

$$
J_{\mathcal{C}_{l+\frac{\lambda}{2}}^{r+\frac{\rho}{2}}(\varsigma)}\left(w^{\bar{q}}\right)-J_{\mathcal{C}_{l+\frac{\lambda}{2}}^{r+\frac{\rho}{2}}(\varsigma)}(\omega) \geq c_{1} \mathcal{H}^{n-1}\left(A_{\bar{q}} \cap B_{r}(\varsigma)\right) .
$$

Proof. Set $R=r+\frac{\varrho}{2}$, then we have $\varphi(\xi)=\bar{q} \phi(|\xi-\varsigma|, R)$ with $\phi(\cdot, R):[0, R] \rightarrow$ $\mathbb{R}$ a positive function which is strictly increasing in $(0, R]$. Moreover we have $\phi(R, R)=1$ and

$$
R_{1}<R_{2}, t \in\left(0, R_{1}\right) \Rightarrow \phi\left(R_{1}-t, R_{1}\right)>\phi\left(R_{2}-t, R_{2}\right) .
$$

Note that $\xi \in B_{r}(\varsigma)$ implies $\varphi(\xi) \leq \bar{q} \phi\left(r, r+\frac{\varrho}{2}\right)$. Therefore for $\xi \in B_{r}(\varsigma) \cap A_{\bar{q}}$ we have

$$
\begin{aligned}
& \mathbf{e}_{l+\frac{\lambda}{2}}\left(\bar{q} v^{w}\right)-\mathbf{e}_{l+\frac{\lambda}{2}}\left(\varphi v^{w}\right)-D_{q} \mathbf{e}_{l+\frac{\lambda}{2}}\left(\varphi v^{w}\right)(\bar{q}-\varphi) \\
= & \int_{\varphi}^{\bar{q}}\left(D_{q} \mathbf{e}_{l+\frac{\lambda}{2}}\left(s v^{w}\right)-D_{q} \mathbf{e}_{l+\frac{\lambda}{2}}\left(\varphi v^{w}\right)\right) d s \\
\geq & c^{2} \int_{\varphi}^{\bar{q}}(s-\varphi) d s=\frac{1}{2} c^{2}(\bar{q}-\varphi)^{2} \geq \frac{1}{2} c^{2} \bar{q}^{2}\left(1-\phi\left(r, r+\frac{\varrho}{2}\right)\right)^{2},
\end{aligned}
$$

where we have also used $(2.7)_{1}$. The corollary follows from this inequality, from (2.51), (2.54) and from the fact that, by (2.71), the last expression in (2.72) is increasing with $r$. Therefore, for $r \geq r_{0}$, for some $r_{0}>0$, we can assume

$$
c_{1}=\frac{1}{2} c^{2} \bar{q}^{2}\left(1-\phi\left(r_{0}, r_{0}+\frac{\varrho}{2}\right)\right)^{2} .
$$

2.3. Estimating $q^{u}(\varsigma)=\|u(\cdot, \varsigma)-\bar{u}(\cdot)\|_{l}$

Let $u$ be as in Theorem 1.4 and $l_{0}, q^{\circ}$ as in Lemma 2.1 and assume that $\varsigma$ is such that

$$
\|u(\cdot, \varsigma)-\bar{u}(\cdot)\|_{l} \geq q^{\circ},
$$

for some $l \geq l_{0}$. Then $u \in C_{S}^{\operatorname{Exp}}\left(\bar{\Omega}, \mathbb{R}^{m}\right)$ implies that there is $r_{0}>0$ independent of $l \geq l_{0}$ such that

$$
\|u(\cdot, \xi)-\bar{u}(\cdot)\|_{l} \geq \bar{q}, \text { for }|\xi-\varsigma| \leq r_{0} .
$$

Let $j_{0} \geq 0$, be minimum value of $j$ that violates the inequality

$$
c_{1} \frac{r_{0}^{n-1}}{2}\left(1+\frac{c_{1}}{C_{1}}\right)^{j} \leq C_{1}\left(\left(r_{0}+(j+1) \varrho\right)^{n-1}-\left(r_{0}+j \varrho\right)^{n-1}\right),
$$


where $c_{1}$ and $C_{1}$ are the constants in Corollary 2.8 and Lemma 2.5. Let $l^{\circ} \geq l_{0}$ be fixed so that

$$
C_{0}\left(r_{0}+j_{0} \varrho\right)^{n-1} e^{-k l^{\circ}} \leq c_{1} \theta_{n-1} \frac{r_{0}^{n-1}}{2},
$$

where $C_{0}$ is defined in Lemma 2.4 and $\theta_{n}$ is the measure of the unit ball in $\mathbb{R}^{n}$,

Proposition 2.9. Let $\lambda, \varrho, \bar{q} \in\left(0, q^{\circ}\right)$ and $l^{\circ} \geq l_{0}$ be fixed as before and let $r^{\circ}=$ $r_{0}+j_{0} \varrho$ where $j_{0} \geq 0$ is the minimum value of $\bar{j}$ that violates $(2.76)$. Assume $l \geq l^{\circ}$ and assume that $\mathcal{C}_{l+\lambda}^{r^{\circ}+2 \varrho}(\varsigma) \subset \Omega$ satisfies

$$
d\left(\mathcal{C}_{l+\lambda}^{r^{\circ}+2 \varrho}(\varsigma), \partial \Omega\right) \geq l+\lambda
$$

Then

$$
q^{u}(\varsigma)=\|u(\cdot, \varsigma)-\bar{u}(\cdot)\|_{l+\frac{\lambda}{2}}<q^{\circ} .
$$

Proof. Suppose instead that

$$
\|u(\cdot, \varsigma)-\bar{u}(\cdot)\|_{l+\frac{\lambda}{2}} \geq q^{\circ}
$$

and set

$$
\sigma_{0}:=\theta_{n-1} \frac{r_{0}^{n-1}}{2} .
$$

Then $l \geq l^{\circ} \geq l_{0}$ and (2.75) imply

$$
\mathcal{H}^{n-1}\left(A_{\bar{q}} \cap B_{r_{0}}(\varsigma)\right) \geq 2 \sigma_{0} .
$$

For each $0 \leq j \leq j_{0}$ let $r_{j}:=r_{0}+j \varrho$ and let $v_{j}, w_{j}, w_{j}^{\bar{q}}$ and $\omega_{j}$ be the maps $v, w, w^{\bar{q}}$ and $\omega$ defined in Lemma 2.4, Lemma 2.5, Lemma 2.6 and Lemma 2.7 with $l \geq l^{\circ}$ and $r=r_{j}$. Then from these Lemmas and Corollary 2.8 we have

$$
\begin{gathered}
J(u)_{\mathcal{C}_{l+\lambda}^{r_{j}+2 \varrho}(\varsigma)}-J\left(v_{j}\right)_{\mathcal{C}_{l+\lambda}^{r_{j}+2 \varrho}(\varsigma)} \geq-C_{0} r_{j}^{n-1} e^{-k l^{\circ}}, \\
J\left(v_{j}\right)_{\mathcal{C}_{l+\lambda}^{r_{j}+2 \varrho}(\varsigma)}-J\left(w_{j}\right)_{\mathcal{C}_{l+\lambda}^{r_{j}+2 \varrho}(\varsigma)} \geq-C_{1} \mathcal{H}^{n-1}\left(A_{\bar{q}} \cap\left(\bar{B}_{\zeta, r_{j+1}} \backslash B_{r_{j}}(\varsigma)\right)\right), \\
J\left(w_{j}\right)_{\mathcal{C}_{l+\lambda}^{r_{j}+2 \varrho}(\varsigma)}-J\left(w_{j}^{\bar{q}}\right)_{\mathcal{C}_{l+\lambda}^{r_{j}+2 \varrho}(\varsigma)} \geq 0, \\
J\left(w_{j}^{\bar{q}}\right)_{\mathcal{C}_{l+\lambda}^{r_{j}+2 \varrho}(\varsigma)}-J\left(\omega_{j}\right)_{\mathcal{C}_{l+\lambda}^{r_{j}+2 \varrho}(\varsigma)} \geq c_{1} \mathcal{H}^{n-1}\left(A_{\bar{q}} \cap \bar{B}_{\zeta, r_{j}}\right) .
\end{gathered}
$$


From this and the minimality of $u$ it follows

$$
\begin{aligned}
0 \geq & -C_{0} r_{j}^{n-1} e^{-k l^{\circ}}-C_{1} \mathcal{H}^{n-1}\left(A_{\bar{q}} \cap\left(\bar{B}_{\zeta, r_{j+1}} \backslash B_{r_{j}}(\varsigma)\right)\right) \\
& +c_{1} \mathcal{H}^{n-1}\left(A_{\bar{q}} \cap \bar{B}_{\zeta, r_{j}}\right) .
\end{aligned}
$$

Define

$$
\sigma_{j}:=\mathcal{H}^{n-1}\left(A_{\bar{q}} \cap B_{r_{j}}(\varsigma)\right)-\sigma_{0}, \text { for } j \geq 1
$$

For $j=0$ the inequality (2.84), using also (2.77), implies

$$
0 \geq-c_{1} \sigma_{0}-C_{1}\left(\sigma_{1}+\sigma_{0}\right)+2 C_{1} \sigma_{0}+2 c_{1} \sigma_{0} \geq c_{1} \sigma_{0}-C_{1}\left(\sigma_{1}-\sigma_{0}\right)
$$

If $j>0$ in a similar way we get

$$
0 \geq-c_{1} \sigma_{0}-C_{1}\left(\sigma_{j-1}-\sigma_{j}\right)+c_{1}\left(\sigma_{j}+\sigma_{0}\right)=c_{1} \sigma_{j}-C_{1}\left(\sigma_{j+1}-\sigma_{j}\right) .
$$

From (2.86) and (2.87) it follows

$$
\sigma_{j} \geq\left(1+\frac{c_{1}}{C_{1}}\right)^{j} \sigma_{0}
$$

and therefore, using also (2.81),

$$
c_{1}\left(1+\frac{c_{1}}{C_{1}}\right)^{j} \theta_{n-1} \frac{r_{0}^{n-1}}{2} \leq C_{1}\left(\sigma_{j+1}-\sigma j\right) \leq C_{1} \theta_{n-1}\left(r_{j+1}^{n-1}-r_{j}^{n-1}\right) .
$$

This inequality is equivalent to (2.76). It follows that, on the basis of the definition of $j_{0}$, putting $j=j_{0}$ in (2.89) leads to a contradiction with the minimality of $u$.

\subsection{Conclusion of the proof of Theorem 1.4: the exponential estimate}

Lemma 2.10. Assume $r>r^{\circ}+2 \varrho$ and $l>l^{\circ}+\lambda$ and assume that $\mathcal{C}_{l}^{r}\left(\varsigma_{0}\right) \subset \Omega$ satisfies

$$
d\left(\mathcal{C}_{l}^{r}\left(\varsigma_{0}\right), \partial \Omega\right) \geq l
$$

Then there are constants $K_{1}$ and $k_{1}>0$ independent of $r>r^{\circ}+2 \varrho$ and $l>l^{\circ}+\lambda$ such that

$$
\left\|u\left(\cdot, \varsigma_{0}\right)-\bar{u}\right\|_{l} \leq K_{1} e^{-k_{1} r}
$$

Proof. From $r>r^{\circ}+2 \varrho$ it follows that $\left|\varsigma-\varsigma_{0}\right| \leq r-\left(r^{\circ}+2 \varrho\right)$ implies

$$
d\left(\mathcal{C}_{l}^{r^{\circ}+2 \varrho}(\varsigma), \partial \Omega\right) \geq l
$$


Therefore we can invoke Proposition 2.9 to conclude that

$$
\|u(\cdot, \varsigma)-\bar{u}\|_{l} \leq q^{\circ}, \text { for }\left|\varsigma-\varsigma_{0}\right| \leq r-\left(r^{\circ}+2 \varrho\right) .
$$

Let $\varphi: B_{r-\left(r^{\circ}+2 \varrho\right)}\left(\varsigma_{0}\right) \rightarrow \mathbb{R}$ be the solution of

$$
\begin{cases}\Delta \varphi=c^{2} \varphi & \text { in } B_{r-\left(r^{\circ}+2 \varrho\right)}\left(\varsigma_{0}\right) \\ \varphi=q^{\circ} & \text { on } \partial B_{r-\left(r^{\circ}+2 \varrho\right)}\left(\varsigma_{0}\right) .\end{cases}
$$

Then we have

$$
\|u(\cdot, \varsigma)-\bar{u}\|_{l} \leq \varphi(\varsigma), \text { for } \varsigma \in B_{r-\left(r^{\circ}+2 \varrho\right)}\left(\varsigma_{0}\right) .
$$

This follows by the same argument leading to (2.65) in the proof of Lemma 2.7. Indeed (2.93) shows that $u$ satisfies the assumptions on $w^{\bar{q}}$ in Lemma 2.7 with $q^{\circ}$ instead of $\bar{q}$ and therefore if (2.95) does not hold, then by proceeding as in the proof of Lemma 2.7 we can construct a competing map $\omega$ that satisfies (2.95) and has less energy than $u$ contradicting its minimality property. In particular (2.95) implies

$$
\left\|u\left(\cdot, \varsigma_{0}\right)-\bar{u}\right\|_{l} \leq \varphi\left(\varsigma_{0}\right) .
$$

On the other hand it can be shown, see Lemma 2.4 in [16], that there is a constant $h_{0}>0$ such that

$$
\phi(0, r) \leq e^{-h_{0} r}, \text { for } r \geq r_{0}
$$

From this and (2.96) we get

$$
\varphi\left(\varsigma_{0}\right)=\bar{q} \phi\left(0, r-\left(r^{\circ}+2 \varrho\right)\right) \leq \bar{q} e^{h_{0}\left(r^{\circ}+2 \varrho\right)} e^{-h_{0} r}=K_{1} e^{-k_{1} r} .
$$

This concludes the proof with $K_{1}=\bar{q} e^{h_{0}\left(r^{\circ}+2 \varrho\right)}$ and $k_{1}=h_{0}$.

We are now in the position of proving the exponential estimate (1.15) in Theorem 1.4. We distinguish two cases:

Case $1 x=(s, \xi) \in \Omega$ satisfies $s>\frac{1}{2} d(x, \partial \Omega)$. In this case, taking also into account that $\Omega$ satisfies (1.7), we have

$$
d\left(x, \partial \Omega^{+}\right) \geq \frac{1}{2} d(x, \partial \Omega) .
$$

From this and Theorem 1.3 it follows

$$
\begin{aligned}
& |u(s, \xi)-\bar{u}(s)| \leq|u(s, \xi)-a|+|\bar{u}(s)-a| \\
& \leq K_{0} e^{-k_{0} d\left(x, \partial \Omega^{+}\right)}+K_{0} e^{-k_{0} s} \leq 2 K_{0} e^{-\frac{1}{2} k_{0} d(x, \partial \Omega)},
\end{aligned}
$$

where we have also used (2.2). 
Case $2 x=(s, \xi) \in \Omega$ satisfies $0 \leq s \leq \frac{1}{2} d(x, \partial \Omega)$. In this case, elementary geometric considerations and the assumption (1.7) on $\Omega$ imply the existence of $\alpha \in(0,1)\left(\alpha=\frac{1}{4}\right.$ will do) such that

$$
\begin{gathered}
\mathcal{C}_{s+\alpha d(x)}^{\alpha d(x)}(\xi) \subset \Omega \text { and } \\
d\left(\mathcal{C}_{s+\alpha d(x)}^{\alpha d(x)}(\xi), \partial \Omega\right) \geq s+\alpha d(x)
\end{gathered}
$$

where we have set $d(x):=d(x, \partial \Omega)$. From (2.100) and Lemma 2.10 it follows

$$
\|u(\cdot, \xi)-\bar{u}\|_{s+\alpha d(x)} \leq K_{1} e^{-k_{1} \alpha d(x)}, \text { for } \alpha d(x)>\max \left\{r^{\circ}+2 \varrho, l^{\circ}+\lambda\right\} .
$$

This and Lemma 2.3 imply

$$
|u(s, \xi)-\bar{u}(s)| \leq C_{2} K_{1}^{\frac{2}{3}} e^{-\frac{2}{3} k_{1} \alpha d(x, \partial \Omega)} .
$$

The exponential estimate follows from (2.99) and (2.102).

\subsection{Proof of Theorem 1.7}

If $\Omega=\mathbb{R}^{n}$ the proof of Theorem 1.4 simplifies since we can avoid the technicalities needed in the case in which $\Omega$ is bounded in the $s=x_{1}$ direction and assume $l=+\infty$. The possibility of working with $l=+\infty$ is based on the following lemma:

Lemma 2.11. Let $u: \mathbb{R}^{n} \rightarrow \mathbb{R}^{m}$ be the symmetric minimizer in Theorem 1.3. Given a smooth open set $O \subset \mathbb{R}^{n-1}$ let $\mathbb{R} \times O$ the cylinder $\mathbb{R} \times O=\{(s, \xi): s \in$ $\mathbb{R}, \xi \in O\}$ and let $W_{0 S}^{1,2}\left(\mathbb{R} \times O, \mathbb{R}^{m}\right)$ be the subset of $W_{S}^{1,2}\left(\mathbb{R} \times O, \mathbb{R}^{m}\right)$ of the maps that vanish on $\partial(\mathbb{R} \times O)$. Then it results

$$
J_{\mathbb{R} \times O}(u) \leq J_{\mathbb{R} \times O}(v)
$$

for each map $v \in u+W_{0 S}^{1,2}\left(\mathbb{R} \times O, \mathbb{R}^{m}\right)$ that satisfies the assumptions in Theorem 1.3.

Proof. Assume there are $\eta>0$ and $v \in u+W_{0 S}^{1,2}\left(\mathbb{R} \times O ; \mathbb{R}^{m}\right)$ that satisfie the assumptions in Theorem 1.3 and the inequality

$$
J_{\mathbb{R} \times O}(u)-J_{\mathbb{R} \times O}(v) \geq \eta .
$$

For each $l>0$ define $\tilde{v} \in u+W_{0 S}^{1,2}\left(\mathbb{R} \times O ; \mathbb{R}^{m}\right)$ by

$$
\tilde{v}= \begin{cases}v & \text { for } s \in[0, l], \text { and } \xi \in O \\ (1+l-s) v+(s-l) u & \text { for } s \in[l, l+1], \text { and } \xi \in O \\ u & \text { for } s \in[l+1,+\infty), \text { and } \xi \in O\end{cases}
$$


The minimality of $u$ implies

$$
\begin{aligned}
0 & \geq J_{[-l-1, l+1] \times O}(u)-J_{[-l-1, l+1] \times O}(\tilde{v}) \\
& =J_{[-l-1, l+1] \times O}(u)-J_{[-l, l] \times O}(v)+\mathrm{O}\left(e^{-k l}\right),
\end{aligned}
$$

where we have also used the fact that both $u$ and $v$ satisfy (1.11). Taking the limit for $l \rightarrow+\infty$ in (2.105) yields

$$
0 \geq J_{\mathbb{R} \times O}(u)-J_{\mathbb{R} \times O}(v)
$$

in contradiction with (2.104).

Once we know that $u$ satisfies (2.103) the same arguments leading to Proposition 2.9 imply the existence of $r^{\circ}>0$ such that

$$
\mathbb{R} \times B_{r^{\circ}}(\xi) \subset \mathbb{R}^{n} \Rightarrow\|u(\cdot, \xi)-\bar{u}\|_{\infty}<q^{\circ},
$$

where $B_{r^{\circ}}(\xi) \subset \mathbb{R}^{n-1}$ is the ball of center $\xi$ and radius $r^{\circ}$. Since the condition $\mathbb{R} \times B_{r^{\circ}}(\xi) \subset \mathbb{R}^{n}$ is trivially satisfied for each $\xi \in \mathbb{R}^{n-1}$ we have

$$
\|u(\cdot, \xi)-\bar{u}\|_{\infty}<q^{\circ}, \text { for every } \xi \in \mathbb{R}^{n-1} .
$$

To conclude the proof we observe that everything that has been said concerning $q^{\circ}$ can be repeated verbatim for each $q \in\left(0, q^{\circ}\right)$. It follows that for each $q \in\left(0, q^{\circ}\right]$ there is a $r(q)>0$ such that (2.106) holds with $q$ in place of $q^{\circ}$ and $r(q)$ in place of $r^{\circ}$. Therefore we have

$$
\|u(\cdot, \xi)-\bar{u}\|_{\infty}<q, \text { for every } \xi \in \mathbb{R}^{n-1} .
$$

Since this holds for each $q \in\left(0, q^{\circ}\right]$ we conclude

$$
u(\cdot, \xi)=\bar{u}, \text { for every } \xi \in \mathbb{R}^{n-1}
$$

which complete the proof of Theorem 1.7.

Theorem 1.6 can also be proved by the method in [6, see Theorem 7.1]. This is due to Lemma 2.1 that allows one to work with infinite cylinders.

\section{Proof of Theorem 1.8}

From an abstract point of view the proof of Theorem 1.8 is essentially the same as the proof of Theorem 1.4 after quantities like $q^{u}$ and $v^{u}$ etc. are reinterpreted and properly redefined in the context of maps equivariant with respect to the group $T$ of the equilateral triangle. We divide the proof into steps pointing out the correspondence with the analogous steps in the proof of Theorem 1.4. We write $x \in \mathbb{R}^{n}$ in the form $x=(s, \xi)$ with $s=\left(s_{1}, s_{2}\right) \in \mathbb{R}^{2}$ and $\xi=\left(x_{3}, \ldots, x_{n}\right) \in \mathbb{R}^{n-2}$. 


\section{Step 1}

From assumption (1.24) in Theorem 1.8 and equivariance it follows

$$
\begin{aligned}
& |u(x)-a| \geq \delta,\left|u(x)-\gamma_{-} a\right|>\delta, \text { for } x \in \gamma_{+} D, d\left(x, \partial \gamma_{+} D\right) \geq d_{0}, \\
& |u(x)-a| \geq \delta,\left|u(x)-\gamma_{+} a\right|>\delta, \text { for } x \in \gamma_{-} D, d\left(x, \partial \gamma_{-} D\right) \geq d_{0}
\end{aligned}
$$

where $\gamma, \pm \gamma$ are defined in the Introduction. From this and assumptions $\mathbf{H}_{3}^{\prime}$ and $\mathbf{H}_{4}^{\prime}$ it follows that we can apply Theorem 1.4 with $\Omega=\left\{\left(s_{1}, s_{2}, \xi\right) \in \mathbb{R}^{n}: s_{1}<0\right\}$ and $a_{ \pm}=\gamma_{ \pm} a$ to conclude that there exist $k, K>0$ such that

$$
\left|u\left(s_{1}, s_{2}, \xi\right)-\bar{u}\left(s_{2}\right)\right| \leq K e^{-k\left|s_{1}\right|}, \text { for } x \in\left\{\left(s_{1}, s_{2}, \xi\right) \in \mathbb{R}^{n}: s_{1}<0\right\} .
$$

In exactly the same way we establish that

$$
\left|\tilde{u}\left(s_{1}, s_{2}\right)-\bar{u}\left(s_{2}\right)\right| \leq K e^{-k\left|s_{1}\right|}, \text { for } s \in\left\{\left(s_{1}, s_{2}\right) \in \mathbb{R}^{2}: s_{1}<0\right\} .
$$

From (3.2), (3.3) and equivariance it follows

$$
|u(s, \xi)-\tilde{u}(s)| \leq K e^{-k|s|}, \text { for } s \in \mathbb{R}^{2} \text {, and } \xi \in \mathbb{R}^{n-2} .
$$

Let $C_{T}^{\operatorname{Exp}}\left(\mathbb{R}^{n}, \mathbb{R}^{m}\right)$ the set of lipshizt maps $v: \mathbb{R}^{n} \rightarrow \mathbb{R}^{m}$ which are equivariant under $T$ and satisfy

$$
\begin{aligned}
& |v(s, \xi)-\tilde{u}(s)| \leq K e^{-k|s|}, \\
& \left|\nabla_{s} v(s, \xi)-\nabla_{s} \tilde{u}(s)\right| \leq K e^{-k|s|}, \text { for } s \in \mathbb{R}^{2}, \text { and } \xi \in \mathbb{R}^{n-2} . \\
& \left|\nabla_{\xi} v(s, \xi)\right| \leq K e^{-k|s|},
\end{aligned}
$$

We remark that from (3.4) we have $u \in C_{T}^{\mathrm{Exp}}\left(\mathbb{R}^{n}, \mathbb{R}^{m}\right)$ for the minimizer $u$ in Theorem 1.8.

\section{Step 2}

Set $B_{l}=\left\{s \in \mathbb{R}^{2}:|s|<l\right\}, l>0$ and let $\langle\cdot, \cdot\rangle_{l}$ denote the standard inner product in $L^{2}\left(B_{l}, \mathbb{R}^{m}\right)$ with associated norm $\|\cdot\|_{l}$.

Set $\|\cdot\|_{1, l}=\|\cdot\|_{W^{1,2}\left(B_{l}, \mathbb{R}^{m}\right)}$.

Note that $u \in C_{T}^{\mathrm{Exp}}\left(\mathbb{R}^{n}, \mathbb{R}^{m}\right)$ implies

$$
\|u(\cdot, \xi)\|_{1, l} \leq C, \text { for } \xi \in \mathbb{R}^{n-2}
$$

for some constant $C>0$.

Define

$$
\begin{aligned}
& \mathcal{B}_{l}^{1,2}=\left\{v \in W_{T}^{1,2}\left(B_{l}, \mathbb{R}^{m}\right): v=0 \text { on } \partial B_{l},\|u(\cdot, \xi)\|_{1, l}<C\right\} \\
& \text { and } \\
& \mathbb{S}=\left\{v \in W_{T}^{1,2}\left(B_{l}, \mathbb{R}^{m}\right): v=0 \text { on } \partial B_{l},\|v\|_{l}=1\right\}
\end{aligned}
$$


In analogy with the definition of $\mathbf{e}_{l}(v)$ in Lemma 2.1, we define the effective potential $\mathbf{E}_{l}(v): \mathcal{B}_{l}^{1,2} \rightarrow \mathbb{R}$ for the case at hand. We set

$$
\begin{aligned}
\mathbf{E}_{l}(v)= & \frac{1}{2}\left(\left\langle\nabla_{s} \tilde{u}+\nabla_{s} v, \nabla_{s} \tilde{u}+\nabla_{s} v\right\rangle_{l}-\left\langle\nabla_{s} \tilde{u}, \nabla_{s} \tilde{u}\right\rangle_{l}\right) \\
& +\int_{B_{l}}(W(\tilde{u}+v)-W(\tilde{u})) d s .
\end{aligned}
$$

From this definition and assumptions $\mathbf{H}_{5}$ and $\mathbf{H}_{6}$, arguing exactly as in the proof of Lemma 2.1, we prove:

Lemma 3.1. Assume $\mathbf{H}_{5}$ and $\mathbf{H}_{6}$. Then there exist $q^{\circ}>0$ and $c>0$ such that

$$
\begin{cases}D_{q q} \mathbf{E}(q v) \geq c^{2} & \text { for } q \in\left[0, q^{\circ}\right], v \in \mathbb{S} \\ \mathbf{E}(q v) \geq \mathbf{E}\left(q^{\circ} v\right) & \text { for } q^{\circ} \leq q, v \in \mathbb{S} \\ \mathbf{E}(q v) \geq \tilde{\mathbf{E}}(p, q, v) & \\ \quad:=\mathbf{E}(p v)+D_{q} \mathbf{E}(p v)(q-p) & \text { for } 0 \leq p<q \leq q^{\circ}, v \in \mathbb{S} \\ D_{p} \tilde{\mathbf{E}}(p, q, v) \geq 0, & \text { for } 0 \leq p<q \leq q^{\circ}, v \in \mathbb{S} .\end{cases}
$$

\section{Step 3}

In Section 2 replace the interval $[-l, l]$ with the ball $B_{l} \subset \mathbb{R}^{2}$, the cylinder $\mathcal{C}_{l}^{r}(\varsigma)$ with the product $B_{l} \times B_{r}(\varsigma), B_{r}(\varsigma) \subset \mathbb{R}^{n-2}$ the ball of center $\varsigma \in \mathbb{R}^{n-2}$ and radius $r$, and observe that, for $O \in \mathbb{R}^{n-2}$ open bounded and smooth, the energy of a map $v \in W_{T}^{1,2}\left(B_{l} \times O, \mathbb{R}^{m}\right)$ can be expressed in the polar form

$$
J_{B_{l} \times O}(v)=\int_{O}\left(\frac{1}{2}\left(\left|\nabla_{\xi} q^{v}\right|^{2}+\left(q^{v}\right)^{2} \sum_{j}\left\langle v_{\xi_{j}}^{v}, v_{\xi_{j}}^{v}\right\rangle_{l}\right)+\mathbf{E}\left(q^{v} v^{v}\right)\right) d \xi,
$$

where $q^{v}$ and $v^{v}$ are defined by

$$
\begin{aligned}
& q^{v}(\xi)=\|v(\cdot, \xi)-\tilde{u}\|_{l}, \quad \text { for } \xi \in O \\
& v^{v}(s, \xi)=\frac{v(s, \xi)-\tilde{u}(s)}{q^{v}(\xi)}, \text { if } q^{v}(\xi)>0 .
\end{aligned}
$$

Then we can follow step by step the arguments in Section 2 to prove statements analogous to Lemmas 2.3-2.7, Corollary 2.8 and establish the analogous of Proposition 2.9. Actually, since we are working in $\mathbb{R}^{n}$ and therefore there is no boundary, the argument simplifies and the condition corresponding to (2.22) in Lemma 2.3, (2.29) in Lemma 2.4 and Proposition 2.9 are not needed. In conclusion, by arguing as in Section 2, we prove that, given $q \in\left(0, q^{\circ}\right]$, there are $l(q)>0$ $r(q)>0$ such that

$$
B_{l} \times B_{r}(\xi) \subset \mathbb{R}^{n}, \text { and } l \geq l(q), r \geq r(q) \Rightarrow q^{u}(\xi)=\|u(\cdot, \xi)-\tilde{u}(\cdot)\|_{l}<q .
$$


Since the condition on the left hand side of (3.10) is trivially satisfied for all $\xi \in$ $\mathbb{R}^{n-2}$ and for all $q \in\left(0, q^{\circ}\right]$ we deduce

$$
u(s, \xi)=\tilde{u}(s), \text { for } s \in \mathbb{R}^{2}, \text { and } \xi \in \mathbb{R}^{n-2}
$$

which concludes the proof.

\section{References}

[1] S. Agmon, "Lectures on Exponential Decay of Solutions of Second Order Elliptic Equations", Math. Notes, Vol. 29, Princeton University, 1982.

[2] G. Alberti, L. Ambrosio and X. CABré, On a long-standing conjecture of E. De Giorgi: simmetry in $3 D$ for general non linearities and a local minimality property, Acta Appl. Math. 65 (2001), 9-33.

[3] N. D. AlikaKos, A new proof for the existence of an equivariant entire solution connecting the minima of the potential for the system $\Delta u-W_{u}(u)=0$, Comm. Partial Differential Equations 37 (2012), 2093-2115.

[4] N. D. Alikakos, On the structure of phase transition maps for three or more coexisting phases, In: "Geometric Partial Differential Equations", M. Novaga and G. Orlandi (eds.), Publications Scuola Normale Superiore, CRM Series, Birkhäuser, 2013.

[5] N. D. AliKaKos and G. FusCo, Entire solutions to equivariant elliptic systems with variational structure, Arch. Ration. Mech. Anal. 202 (2011), 567-597.

[6] N. D. Alikakos and G. FusCo, Density estimates for vector minimizers and applications, Discrete Contin. Dyn. Syst. 35 (2015), 5631-5663.

[7] M. T. BARLOW, R. F. BASS and C. GUI, The Liouville property and a conjecture of De Giorgi, Comm. Pure Appl. Math. 53 (2000), 1007-1038.

[8] H. BeRESTYCKI, L. CAFFARELli and L. NiRENBERG, Monotonicity for elliptic equations in unbounded Lipshitz domains, Comm. Pure Appl. Math. 50 (1997), 1089-1111.

[9] H. Berestycki, L. CAFfarelli and L. Nirenberg, Symmetry for elliptic equations in a half space, In: "Boundary Value Problems for Partial Differential Equations and Applications", J. L. Lions et al. (eds.) RMA Res. Notes Appl. Math. Masson, Paris, 1993, $27-42$.

[10] L. BRONSARD, C. GUI and M. SchatZMAN, A three-layered minimizer in $\mathbb{R}^{2}$ for a variational problem with a symmetric three-well potential, Comm. Pure Appl. Math. 49 (1996), $677-715$.

[11] M. EFENDIEV and F. HAMEL, Asymptotic behavior of solutions of semilinear elliptic equations in unbounded domains: two approaches, Adv. Math. 228 (2011), 1237-1261.

[12] D. JERISON and R. MONNEAU, The existence of a symmetric global minimizer on $R^{n-1}$ implies the existence of a counter-example to a conjecture of De Giorgi in $R^{n}, \mathrm{C} . \mathrm{R}$. Acad. Sci. Paris 333 (2001), 427-431.

[13] A. FARINA, Symmetry for solutions of semilinear elliptic equations in $\mathbb{R}^{N}$ and related conjectures, Atti Accad. Naz. Lincei, Cl. Sci. Fis. Mat. Nat. IX Ser., Rend. Lincei, Mat. Appl. 9 (1999), 255-265.

[14] G. FusCO, Equivariant entire solutions to the elliptic system $\Delta u=W_{u}(u)$ for general G-invariant potentials, Calc. Var. Partial Differential Equations 49 (2013), 963-985.

[15] G. FusCO, On some elementary properties of vector minimizers of the Allen-Cahn energy, Comm. Pure Appl. Anal. 13 (2014), 1045-1060.

[16] G. Fusco, F. LeOnetTi and C. PignotTi, A uniform estimate for positive solutions of semilinear elliptic equations, Trans. Amer. Math. Soc. 363 (2011), 4285-4307.

[17] B. GidAs, W. N. Ni and L. NirenberG, Symmetry and related properties via the maximum principle, Comm. Math. Phys. 68 (1979), 209-243. 
[18] C. Gui and M. Schatzman, Symmetric quadruple phase transitions, Indiana Univ. Math. J. 57 (2008), 781-836.

[19] J. E. Humphreys, "Reflection groups and Coxeter groups", Cambridge Studies in Advanced Mathematics, Vol. 29, Cambridge Univ. Press, 1990, xii + 204 pp.

[20] W. Rudin, "Functional Analysis", McGraw-Hill Series in Higher Mathematics, MacGrawHill Book Co., New York-Düsseldorf-Johannesburg, 1973, xii + 397 pp.

[21] O. SAVIN, Minimal surface and minimizers of the Ginzburg-Landau energy, In: "Symmetry for Elliptic PDEs", Contemp. Math., Vol. 528, Amer. Math. Soc., Providence, RI (2010), $43-58$.

[22] B. WHITE “Topics in GMT”, Lecture Notes by Otis Chodosh, Stanford, 2012.

Department of Mathematics

University of Athens

Panepistemiopolis

15784 Athens, Greece

nalikako@math.uoa.gr

Università de L'Aquila

Via Vetoio (Coppito 2)

67010 Coppito, L' Aquila, Italia

fusco@univaq.it 